\title{
Comparative Assessment of Frequency Dependent Joint Properties Using Direct and Inverse Identification
}

\section{Methods}

\author{
Thesis
}

Presented in Partial Fulfillment of the Requirements for the Degree Masters of Science in the Graduate School of The Ohio State University

By

Benjamin Michael Joodi, B.S

Graduate Program in Mechanical Engineering

The Ohio State University

2014

Thesis Committee:

Rajendra Singh, Advisor

Scott Noll

Brian Harper 


\section{Copyright by}

Benjamin Michael Joodi

2014 


\begin{abstract}
Elastomeric joints are utilized in many automotive applications, and exhibit frequency and excitation amplitude dependent properties. Commercial methods identify only cross-point joint property using displacement excitation at stepped single frequencies. This process is often time consuming and is limited to measuring a single dynamic stiffness term of the joint stiffness matrix. This study focuses on developing tractable laboratory inverse experiments to identify frequency dependent stiffness matrices up to $1000 \mathrm{~Hz}$. Direct measurements are performed on a commercial elastomer test system and an inverse experiment consisting of an elastic beam (with a square cross section) attached to a cylindrical elastomeric joint. The experimental methods are applied to two different elastomeric materials of the same geometry. Sources of error in the inverse methodology are thoroughly examined and explained through simulation which include illconditioning of matrices and the sensitivity to modeling error. The identified translational dynamic stiffness and loss factor values show good agreement between the two identification methods, though challenges remain for the rotational and coupling stiffness terms.
\end{abstract}




\section{Dedication}

I dedicate this thesis to my parents. Without their continuous support, I would have never been able to complete my master's degree. 


\section{Acknowledgements}

I would like to thank Professor Rajendra Singh, Dr. Scott Noll, and Dr. Jason Dreyer for the opportunity and guidance while working on this project. My fellow students of the Acoustics and Dynamics Laboratory were consistently willing to give useful feedback and advice. The undergraduate machine shop was more than helpful as I created laboratory experimental fixtures.

Lastly, I would like to thank Professor Harper for being a member of my thesis defense committee. 


\section{Vita}

August 2013 to Present ...........................BS/MS Program, Mechanical Engineering, The Ohio State University

June 2013 ..............................................S. Program, Mechanical Engineering, The Ohio State University

August 2013 .......................................... Graduate Research Associate,

Department of Mechanical \& Aerospace Engineering The Ohio State University

\section{Publications}

Joodi, B. Examination of Tuning Concepts Using Rubber Mounts. Undergraduate Honors Thesis, The Ohio State University, 2013, https://kb.osu.edu.

\section{Fields of Study}

Major Field: Mechanical Engineering

Concentration: Automobile Noise, Vibration, and Harshness; System Dynamics 


\section{Table of Contents}

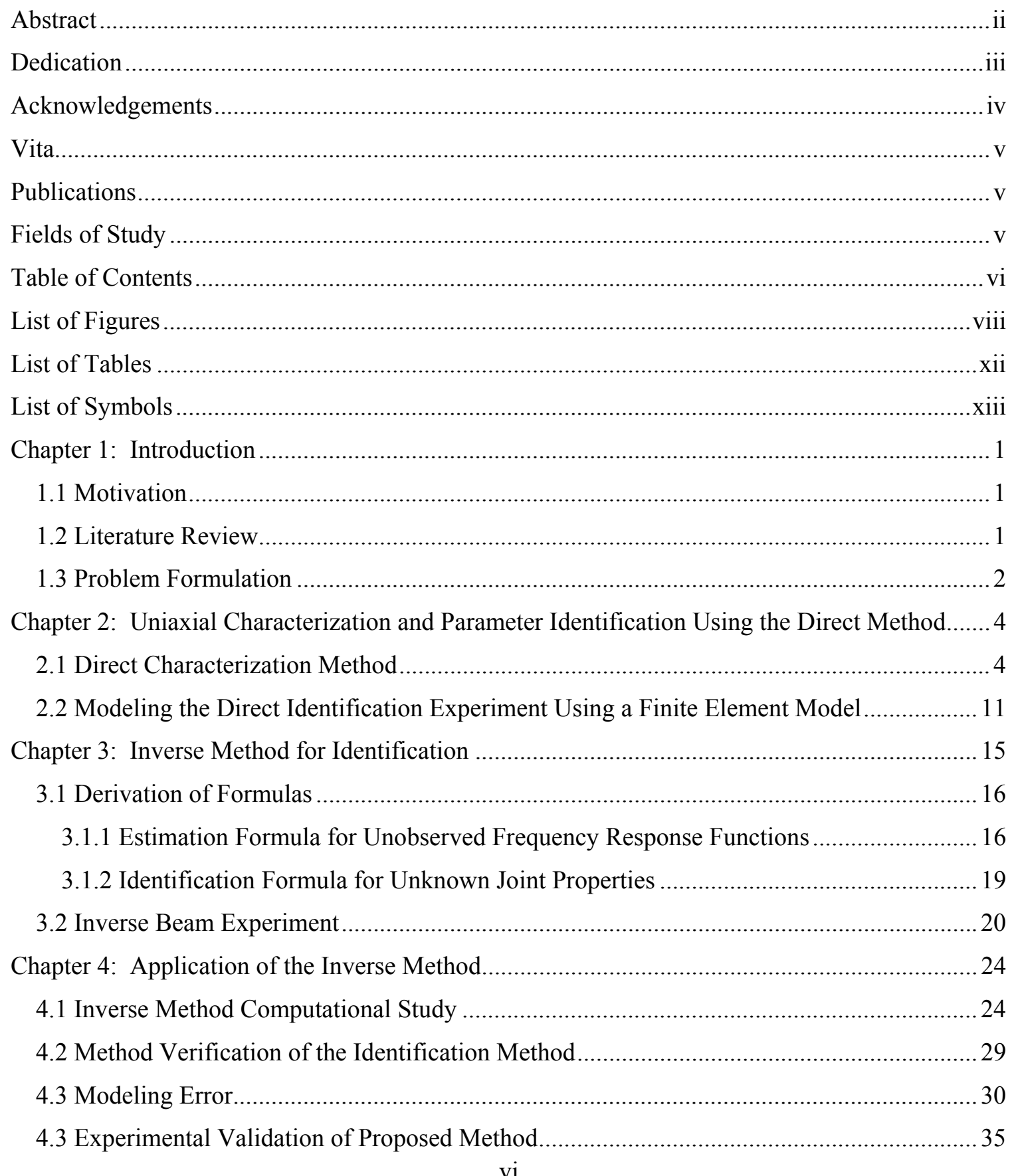


Chapter 5: Reduced Order Models to Include Internal Joint Resonance ..................................... 40

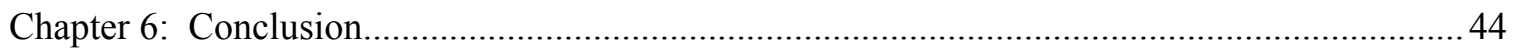

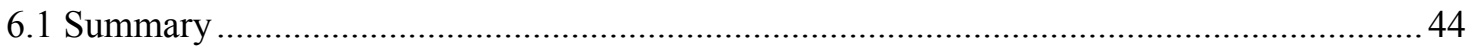

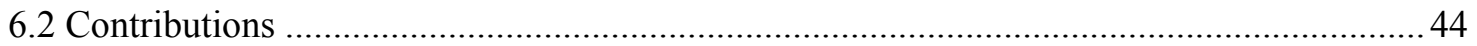

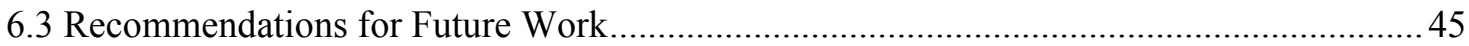

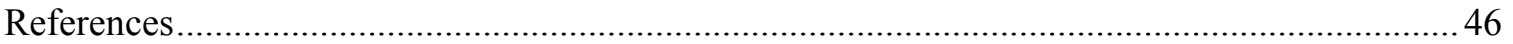

Appendix A: Static Force Deflection Curves for Joints.............................................................. 48 


\section{List of Figures}

Figure 1: Simple beam experiment. (a) Examples analyzed in Hong et al. papers and (b)

Examples yet to be studied. 2

Figure 2: Direct characterization of Elastomeric Joint, (a) Typical joint used in experiments, (b) joint schematic with coordinate system, (c) CAD Model of direct testing experimental fixture with labeled aluminum blocks, (d) simplified equivalent model of direct fixture with X1 associated with displacement of block four and F2 associated with the force seen by the elastomer test system's load cell, (e) finite element model of direct fixture and (f) finite element model of joint fixed on one end and a free boundary condition on the opposing end.

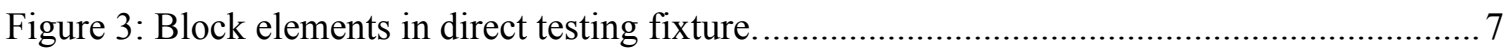

Figure 4: Front view of finite element model of direct experiment. (a) Full fixture assembly. (b)

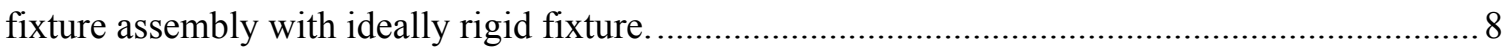

Figure 5: Cross point stiffness-magnitudes as predicted by finite element models in Figure 4.

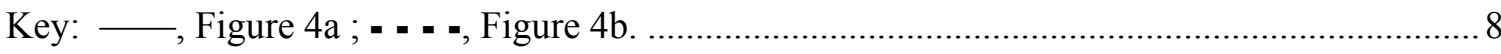

Figure 6: Measured cross-point dynamic stiffness. Magnitude in $(a, b)$ and loss factor in $(c, d)$. Key: (Material A) $૦ \circ \circ \circ, 0.05 \mathrm{~mm}$; *****, $0.1 \mathrm{~mm}$ displacement amplitude (p-p); (Material B)

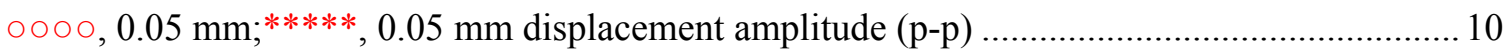

Figure 7: FEA approximation of experimental cross point dynamic stiffness magnitude (a, c) and

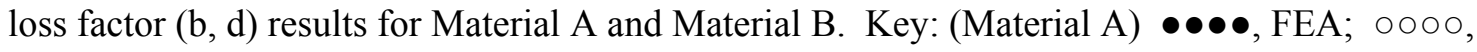

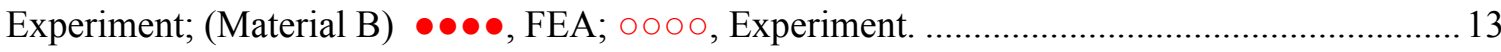


Figure 8: Identified results for cross point dynamic stiffness magnitude (a), driving point dynamic stiffness magnitude (b), cross point loss factor (c), and driving point loss factor (d) for joint finite

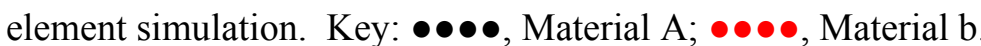

Figure 9: Simplified illustration of the identification method and the corresponding dynamic

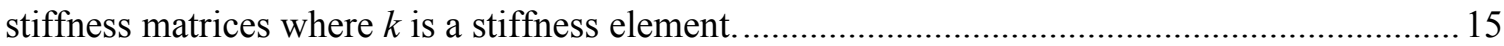

Figure 10: Illustration of important coordinates relevant to the identification method.

Figure 11: Inverse beam models showing (a) conceptual model of constrained structure, (b) conceptual model of unconstrained structure and (c) finite element model of unconstrained structure

Figure 12: Conditioning of $\boldsymbol{D}_{\bar{n} \bar{m}}^{o}(\mathrm{a}), \boldsymbol{H}_{n e}^{*}$ (b), and $\boldsymbol{H}_{m n}^{o}$ (c) for Models 1 and Model 2. Key: - - --, Model 1; - Model 2 .26

Figure 13: FEA identified translational dynamic stiffness magnitude (b) and loss factor (c) for Model 1 displaying effect of conditioning of $\boldsymbol{D}_{\overline{\bar{m}} \bar{m}}^{o}$ (a)

Figure 14: FEA identified translational dynamic stiffness magnitude (b) and loss factor (c) for Model 2 displaying effect of conditioning of $\boldsymbol{D}_{\bar{\pi} \bar{m}}^{o}$ (a) 28

Figure 15: Verification of inverse stiffness magnitude and loss factor for Material A showing (a, b) Translational, $(\mathrm{c}, \mathrm{d})$ coupling, and $(\mathrm{e}, \mathrm{f})$ rotational stiffness components: Key: —— Inverse; - - -, Direct.

Figure 16: Inverse stiffness magnitude and loss factor for varied elastic modulus of unconstrained structure. $(\mathrm{a}, \mathrm{b})$ Translational, $(\mathrm{c}, \mathrm{d})$ coupling, and $(\mathrm{e}, \mathrm{f})$ rotational components: Key: $=-=-, \eta_{o}=\eta * / 3 ;=-=, \eta_{o}=2 \eta * / 3 ;=-=-, \eta_{o}=\eta * ;=-=-, \eta_{o}=4 \eta * / 3 ;=-\cdots, \eta_{o}=5 \eta * / 3.32$ ix 
Figure 17: Inverse stiffness magnitude and loss factor for varied density of unconstrained structure. $(\mathrm{a}, \mathrm{b})$ Translational, $(\mathrm{c}, \mathrm{d})$ coupling, and $(\mathrm{e}, \mathrm{f})$ rotational components: Key: $===-\rho_{o}=$ $.98 \rho * ;==-=, \rho_{o}=.99 \rho * ;=-=-, \rho_{o}=\rho *,==-\rho_{o}=1.01 \rho * ;=-=, \rho_{o}=1.02 \rho *$.

Figure 18 Inverse stiffness magnitude and loss factor for varied elastic modulus of unconstrained structure. (a, b) Translational, $(\mathrm{c}, \mathrm{d})$ coupling, and $(\mathrm{e}, \mathrm{f})$ rotational components: Key: $===-E_{o}$

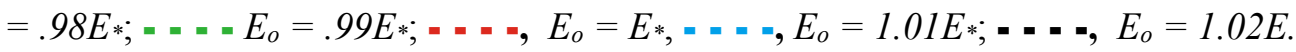

Figure 19: Mass effect due to accelerometer on calculated frequency response functions. (a) Driving point compliance response at coordinate 2 and (b) absolute error. Key:,- 1 accelerometer attached to constrained system; —_ 7 accelerometers attached to constrained system. 36

Figure 20: Dynamic displacement of joint (enlarged deformation). 37

Figure 21: Comparison of inverse FEA and experimental identified dynamic stiffness magnitude (a, b) and loss factor (c, d). Key: ——, Material A Experiment . . . - Material A FEA; ——,

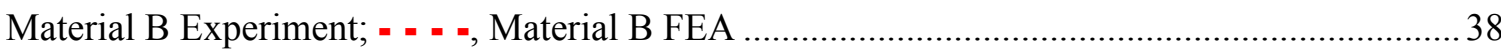

Figure 22: Comparisons of direct and inverse results for low frequency identified $(a, b)$ dynamic stiffness magnitude and (c, d) loss factor. Key: (Material A) ——, Inverse Experiment; - - - - , Direct Experiment (Material B) ——, Inverse Experiment; - - - - , Direct Experiment

Figure 23: Lumped models to approximate joint behavior for a (a) single degree of freedom approximation and (b) two degree of freedom approximation.

Figure 24: Comparison of lumped parameter approximations and inverse FEA showing the $(a, b)$ translational stiffness magnitude (c, d) translational loss factor. Key: , — Material A Inverse 
FEA; . . . -, Single Degree of Freedom Model; ___, Material B Inverse FEA; - . . -, Two Degree

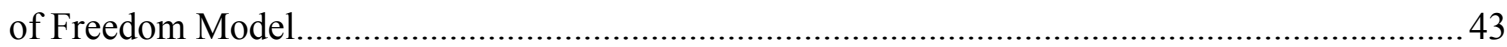

Figure 25: Static force deflection curves for two joints of different elastic materials. Key: (a) -

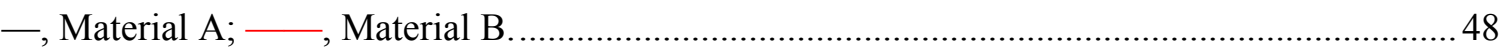




\section{List of Tables}

Table 1: Difference between $0.05 \mathrm{~mm}$ and $0.1 \mathrm{~mm}$ displacement excitation tests checking for

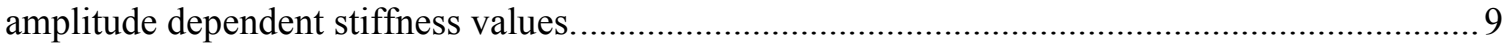

Table 2: Identified material properties and parameters of the two materials ................................. 12

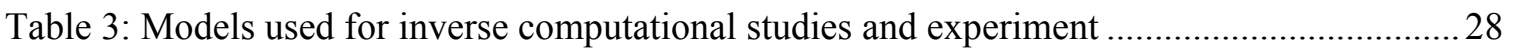

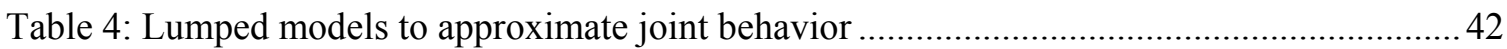




\section{List of Symbols}

\begin{tabular}{|c|c|}
\hline Symbols & 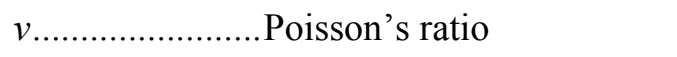 \\
\hline A ............................ & 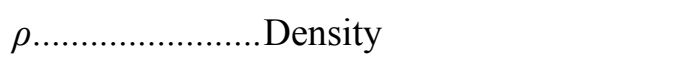 \\
\hline C..................... Viscous damping matrix & $\sigma \ldots \ldots \ldots \ldots \ldots \ldots \ldots$ Singular value \\
\hline D...................... Dynamic stiffness matrix & 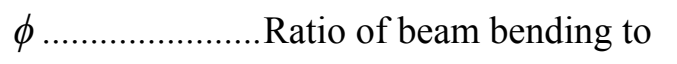 \\
\hline E ......................... Elastic modulus & Shear stiffness \\
\hline$f \ldots \ldots \ldots \ldots \ldots \ldots \ldots . . .$. Frequency & 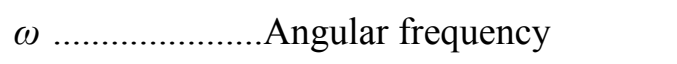 \\
\hline$F \ldots \ldots \ldots \ldots \ldots \ldots \ldots \ldots$ Force & Subscripts \\
\hline $\boldsymbol{F} \ldots \ldots \ldots \ldots \ldots \ldots \ldots \ldots \ldots$ Force vector & e ......................Excited location \\
\hline G...................... Modulus of rigidity & 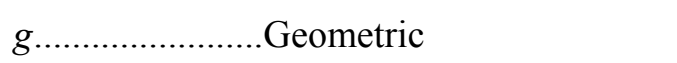 \\
\hline $\boldsymbol{H}$...................... Compliance matrix & $j \ldots \ldots \ldots \ldots \ldots \ldots \ldots \ldots \ldots$ Joint \\
\hline 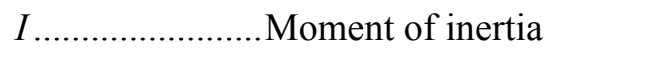 & m........................Non-observed location \\
\hline$I \ldots \ldots \ldots \ldots \ldots \ldots \ldots$ Identity matrix & 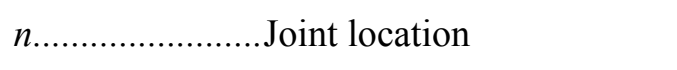 \\
\hline 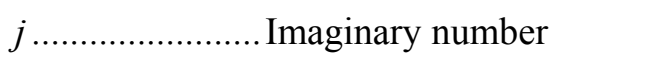 & $N \ldots \ldots \ldots \ldots \ldots \ldots \ldots . . .$. Total number of locations \\
\hline$k \ldots \ldots \ldots \ldots \ldots \ldots \ldots . .$. Dynamic stiffness & o.....................Freely suspended model \\
\hline 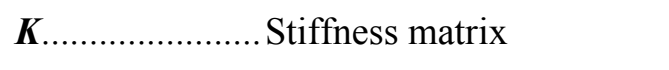 & u.....................nknown properties \\
\hline$L \ldots \ldots \ldots \ldots \ldots \ldots \ldots . .$. Length & x.......................... Translational displacement \\
\hline m......................Mass & 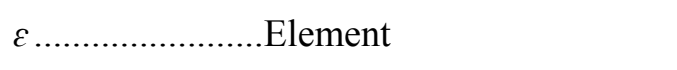 \\
\hline M......................Mass matrix & 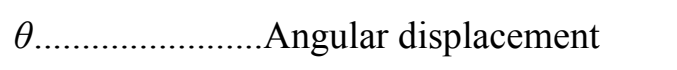 \\
\hline 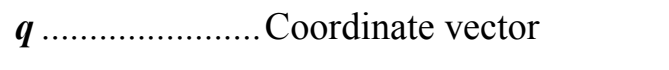 & 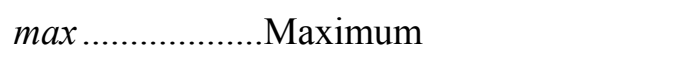 \\
\hline 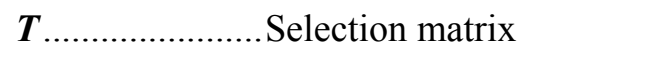 & 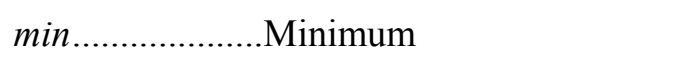 \\
\hline$X \ldots \ldots \ldots \ldots \ldots \ldots \ldots$ Displacement & Superscripts \\
\hline$\kappa \ldots \ldots \ldots \ldots \ldots \ldots \ldots . . . . . . \ldots$ Condition number & Real part \\
\hline$\lambda \ldots \ldots \ldots \ldots \ldots \ldots \ldots . .$. Shear correction factor & ".....................Imaginary part \\
\hline
\end{tabular}


*....................... Constrained model

- .......................... cot connected to

$a$.....................Auxiliary model

c......................... Condensed

L ........................eft inverse
$R$....................Right inverse

$t$..................... Transpose

〜............................ 


\section{Chapter 1: Introduction}

\subsection{Motivation}

Elastomeric joints are commonly used to connect two substructures providing isolation and/or motion control to a system. Current methods used to identify joint properties commonly employ a cross-point measurement where a uniaxial displacement excitation is applied to the joint component under preload $[1,2]$. This method requires the component to be tested with a specified input displacement or force amplitude in a step-sine sweep fashion $[2,3]$. Typical machines used for these measurements consist of commercial elastomer test systems [4] where its actuator excites the joint with a displacement input and measures the force response with a load cell $[2,3]$. These measurements are often limited frequency range (less than $1000 \mathrm{~Hz}$ ) testing due to resonances and capabilities of the machines $[1,2]$. Due to these characteristics common to direct measurements, the test can often be time consuming and only able to determine single stiffness terms in the joint stiffness matrix. Inverse methods have been developed as another approach to characterize joint stiffness properties [1, 5-7].

\subsection{Literature Review}

Kim and Singh [1], incorporate two inertial elements connected to each end of a joint to identify frequency dependent dynamic stiffness matrices using the measured mobilities of the

overall system and rigid body system theory. This method shows promising results in identifying high frequency stiffness properties of the joint; however, the experimental configuration is not capable of incorporating preload [1]. 
Other methods [5-7] incorporate the use of elastic beam theory in place of the rigid inertia elements and thus permit incorporating preload into the identification experiment. Hong and Lee [6] develop a method in which an unconstrained structure (base structure) and an experimental setup (base structure connected to joints) is used to identify joint properties. By acquiring the complete dynamic compliance matrices of the two models, the identified joint properties can be found by subtraction of the inverse of the two compliance matrices [6]. This method appears useful to identify multidimensional frequency dependent properties under a variety of preload conditions [6]. Although such formulations exist in open literature, this study serves to fill the void of a lack of experimental validation where an elastic beam element is incorporated in the setup to identify frequency dependent properties of elastomeric joints.

Examples analyzed

(a)

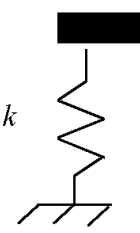

$k$

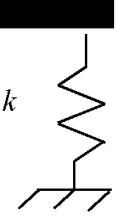

(b)

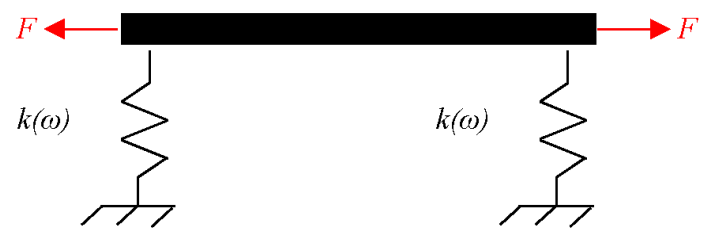

Figure 1: Simple beam experiment where $k$ is stiffness, $F$ is force and $\omega$ is frequency (a) Examples analyzed in Hong et al. [6, 7] papers and (b) Examples yet to be studied.

\subsection{Problem Formulation}

This study extends the prior work of Hong and Lee [6] by conducting benchmark experiments that contain joints with frequency dependent properties. The joints considered are 
composed of a cylindrical elastomeric material with steel caps at each end. Two joints of different material are analyzed. The scope is focused on identifying results in the frequency domain up to $1000 \mathrm{~Hz}$.

The specific objectives of this study are as follows: 1) Design tractable laboratory experiments that allow for a comparison between inverse and direct identification methods; 2) Conduct numerical verification simulations to examine sources of error common to the inverse approach; and 3) Conduct benchmark validation experiments to identify the joint properties; and 4) Develop reduced order lumped parameter joint models The experimental validation study intentionally examines only the translational stiffness term and leaves the rotational and coupling terms for future research. 


\section{Chapter 2: Uniaxial Characterization and Parameter Identification Using the Direct Method}

\subsection{Direct Characterization Method}

The joint is a symmetric cylindrical elastomeric material with steel caps on opposing ends as illustrated in Figure 2a and $\mathrm{b}$. The joint rotational, $\theta_{1}$ and $\theta_{2}$, and translational, $X_{1}$ and $X_{2}$, degrees of freedom are considered; thus, the joint stiffness matrix is given as

$$
\left[\begin{array}{c}
F_{1} \\
M_{1} \\
F_{2} \\
M_{2}
\end{array}\right]=\left[\begin{array}{cccc}
\tilde{k}_{F_{1} X_{1}} & \tilde{k}_{F_{1} \theta_{1}} & \tilde{k}_{F_{1} X_{2}} & \tilde{k}_{F_{1} \theta_{2}} \\
\tilde{k}_{M_{1} X_{1}} & \tilde{k}_{M_{1} \theta_{1}} & \tilde{k}_{M_{1} X_{2}} & \tilde{k}_{M_{1} \theta_{2}} \\
\tilde{k}_{F_{2} X_{1}} & \tilde{k}_{F_{2} \theta_{1}} & \tilde{k}_{F_{2} X_{2}} & \tilde{k}_{F_{2} \theta_{2}} \\
\tilde{k}_{M_{2} X_{1}} & \tilde{k}_{M_{2} \theta_{1}} & \tilde{k}_{M_{2} X_{2}} & \tilde{k}_{M_{2} \theta_{2}}
\end{array}\right]\left[\begin{array}{c}
X_{1} \\
\theta_{1} \\
X_{2} \\
\theta_{2}
\end{array}\right]
$$

where $F_{1}$ and $F_{2}$ are applied forces, and $M_{1}$ and $M_{2}$ are the moments. The stiffness terms of interest to identify consist of the translational stiffness element, $\tilde{k}_{F_{1} X_{1}}$, the rotational stiffness element, $\tilde{k}_{M_{1} \theta_{1}}$, and the coupling terms, $\tilde{k}_{F_{1} \theta_{1}}$, and $\tilde{k}_{M_{1} X_{1}}$.

Dynamic stiffness, at a given frequency, of a damped joint is defined as

$$
\begin{gathered}
\tilde{k}=k^{\prime}+j k^{\prime \prime}, \\
\eta=\frac{k^{\prime \prime}}{k^{\prime}},
\end{gathered}
$$


where $k^{\prime}$ is the real (storage) component of the dynamic stiffness, $k^{\prime \prime}$ is the imaginary component (dissipative) of the dynamic stiffness, $j$ is $\sqrt{-1}$, and $\eta$ is the loss factor- defined by Equation (3).

The joints are tested on an elastomer test system [2-4] which is a non-resonant test where input displacement is applied at one end of the specimen, and the force transmitted is measured at the other end. Since the stiffness of the joint is being pursued, the fixture itself utilized for experimentation must be stiff relative to the joint [8]. Accordingly, aluminum is chosen as the fixture material. The fixture is composed of six blocks bolted together, as illustrated in Figure 2c. The bottom fixture is composed of blocks labeled 1, 2 and 3, and block 3 is mounted to the load cell of the test system. The top fixture, composed of block 4, provides an input displacement $\left(X_{I}\right)$ to the joint which transmits a force to blocks $1,2,3$ and the load cell of the test system $\left(F_{2}\right)$. The direct test measures $\tilde{k}_{F_{2} X_{1}}$, which is a cross-point term of the stiffness matrix Equation (1). 
(a)

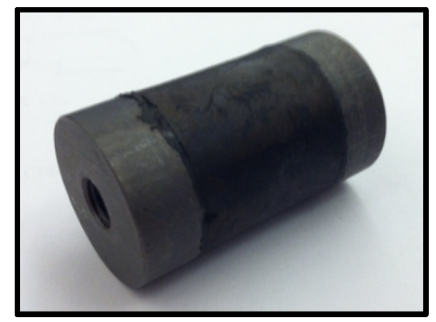

(c)

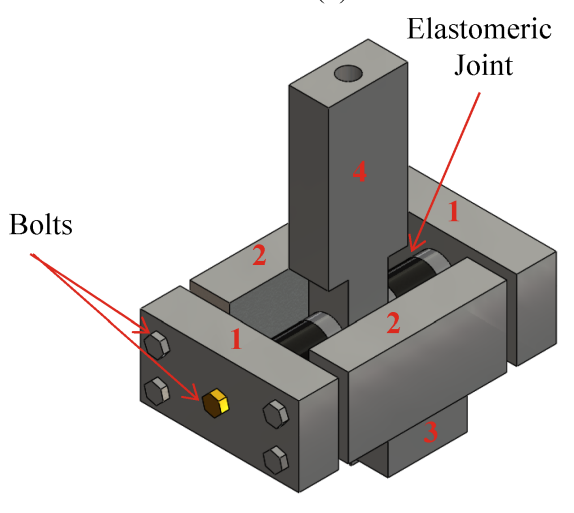

(e)

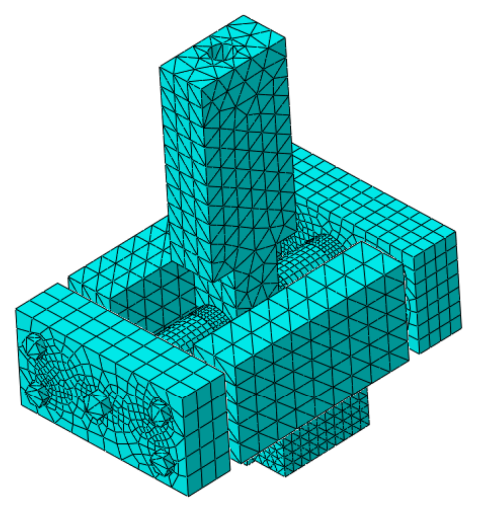

(b)

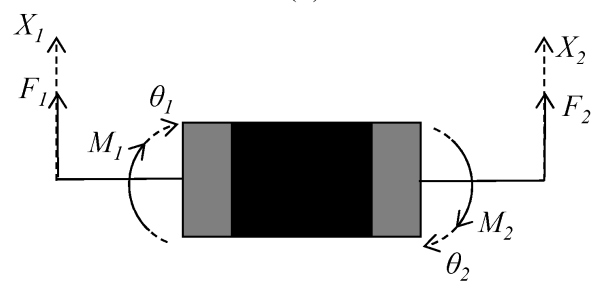

(d)

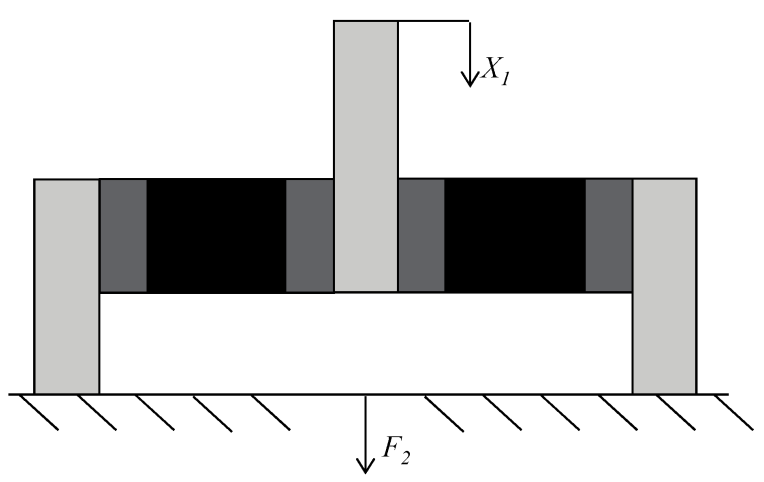

(f)

Figure 2: Direct characterization of Elastomeric Joint, (a) typical joint used in experiments, (b) joint schematic with coordinate system, (c) CAD Model of direct testing experimental fixture with labeled aluminum blocks, (d) simplified equivalent model of direct fixture with $X_{1}$ associated with displacement of block four and $F_{2}$ associated with the force seen by the elastomer test system's load cell, (e) finite element model of direct fixture and (f) finite element model of joint fixed on one end and a free boundary condition on the opposing end. 


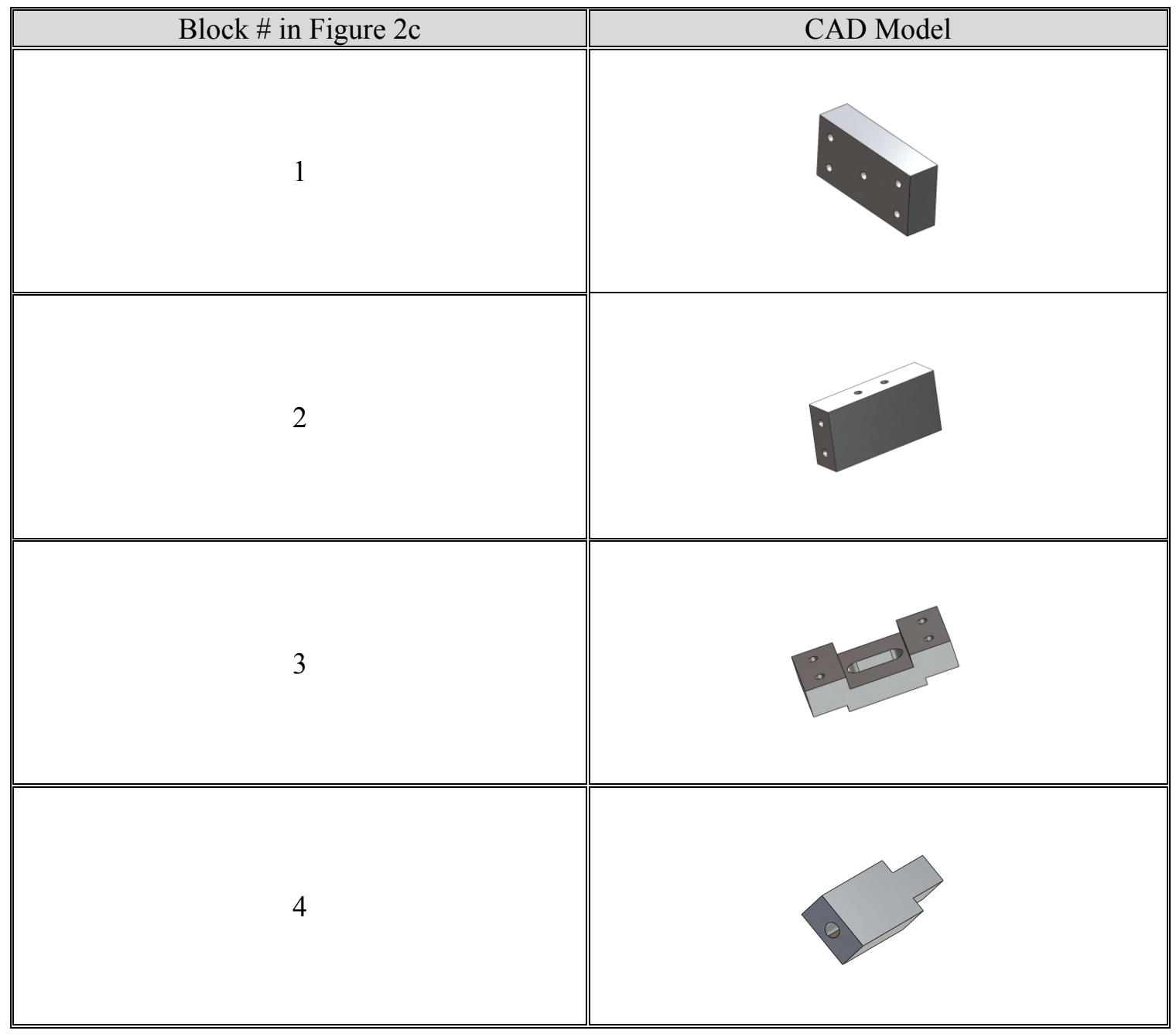

Figure 3: Block elements in direct testing fixture.

It is important to determine whether the fixture has dynamics that may affect the identified joint stiffness results. A direct dynamic test is simulated to an FEA model of the fixture and joints (Figure 2e). A vertical harmonic translational displacement at $X_{1}$ and the response force is measured at location $F_{2}$ (Figure 2d). First, a simulation is completed on the complete assembled 
fixture, and again on the assembly without the bottom fixture elements, as shown in Figure 4a and 4b. In order to develop Figure $4 \mathrm{~b}$, constraints are applied to the bushing ends kinematically coupling the degrees of freedom to the test system load cell. Joint elastomeric material properties are chosen with a density of $1300 \mathrm{~kg} / \mathrm{m}^{3}$ and an elastic modulus of $15.38 \mathrm{MPa}$ (properties of Material A discussed later in this section). The dynamic stiffness magnitude of the two FEA models shown on Figure 5 agree reasonably well, signifying that the dynamics of the fixture is beyond the frequency range of interest, and this fixture does not affect the identified stiffness results.

(a)

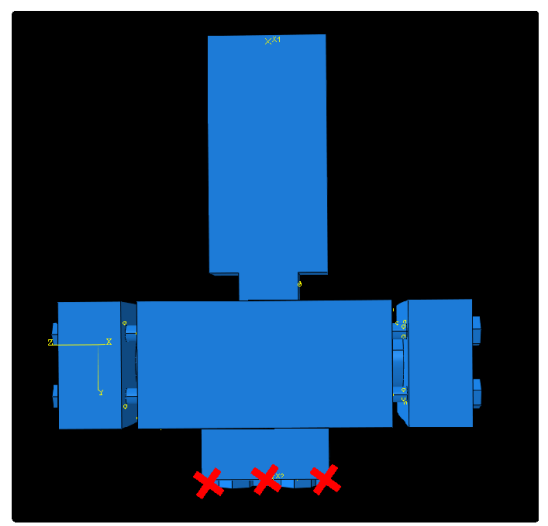

(b)

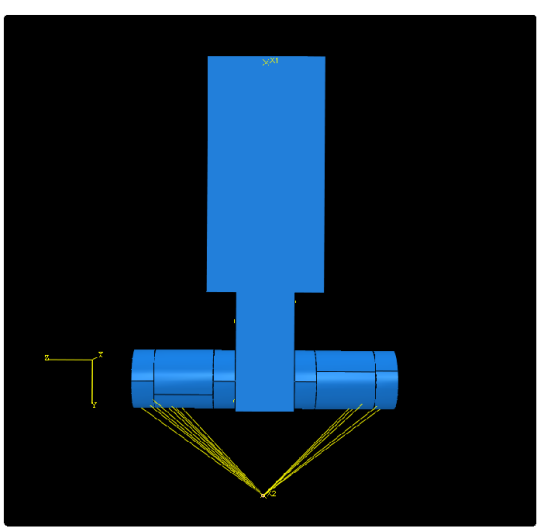

Figure 4: Front view of finite element model of direct experiment. (a) Full fixture assembly, and (b) Fixture assembly with ideally rigid fixture.

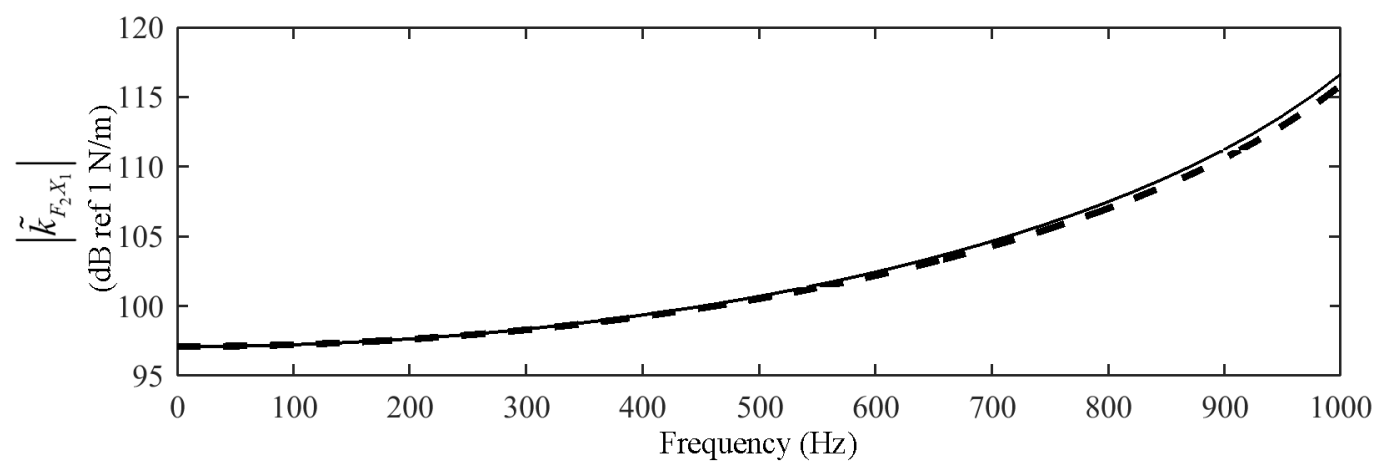

Figure 5: Cross point stiffness-magnitudes as predicted by finite element models in Figure 4. Key: —, Figure 4a; - - - -, Figure 4b. 
The experimental test consists of a cross point measurement up to $600 \mathrm{~Hz}$ with a frequency resolution of $25 \mathrm{~Hz}$. The upper frequency in the test is $600 \mathrm{~Hz}$ rather than $1000 \mathrm{~Hz}$ due to a limitation in the elastomer test system as it failed to control to the specified displacement amplitude beyond $600 \mathrm{~Hz}$. The displacement amplitude is chosen to create a sine wave that is within 5\% of its amplitude peak. The test is performed with two input displacement peak to peak amplitudes, $0.05 \mathrm{~mm}$ and $0.1 \mathrm{~mm}$. The two excitation amplitudes are chosen to examine the amplitude dependent property of the elastomer joint. Figure 6 and Table 1 show that the differences between the $0.05 \mathrm{~mm}$ and $0.1 \mathrm{~mm}$ amplitude tests are all less than $6 \%$. Since smaller amplitudes closer resemble impulse hammer excitation experiments, the $0.05 \mathrm{~mm}$ displacement amplitude is chosen for further comparisons with inverse identification results.

Table 1: Difference between $0.05 \mathrm{~mm}$ and $0.1 \mathrm{~mm}$ displacement excitation tests checking for amplitude dependent stiffness values.

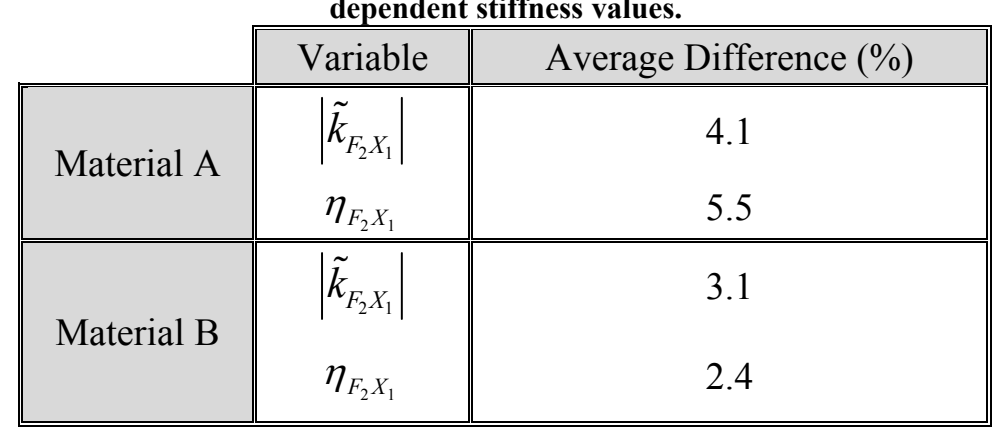


(a)

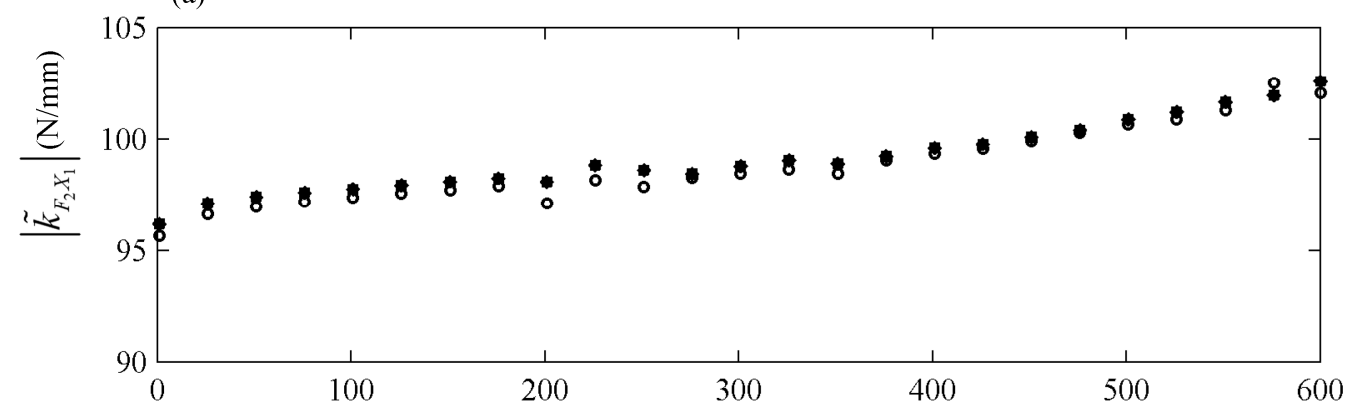

(b)

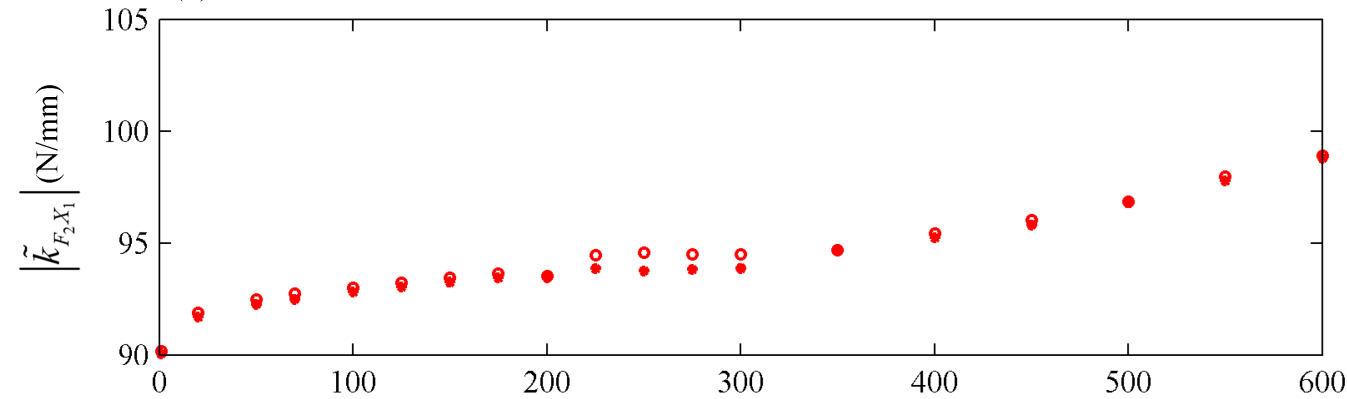

(c)

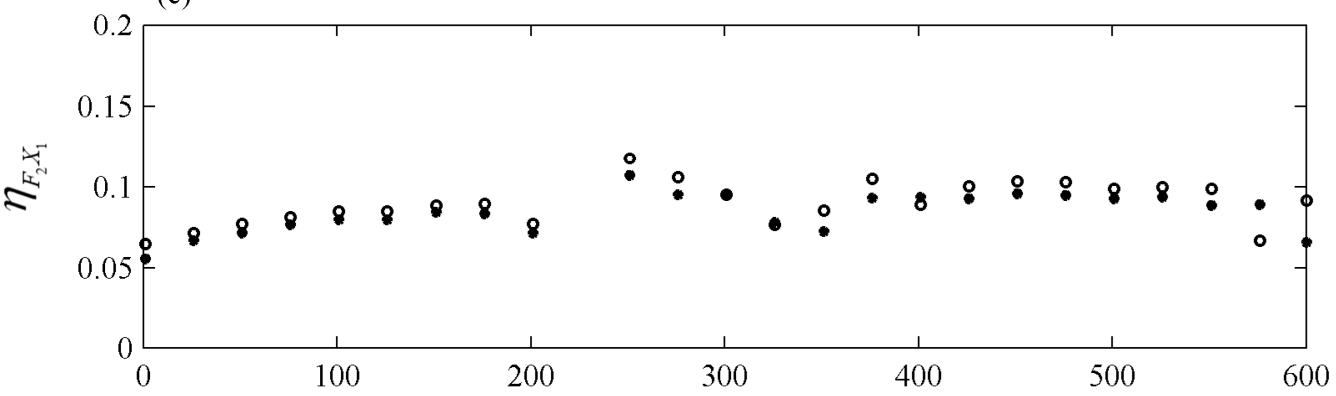

(d)

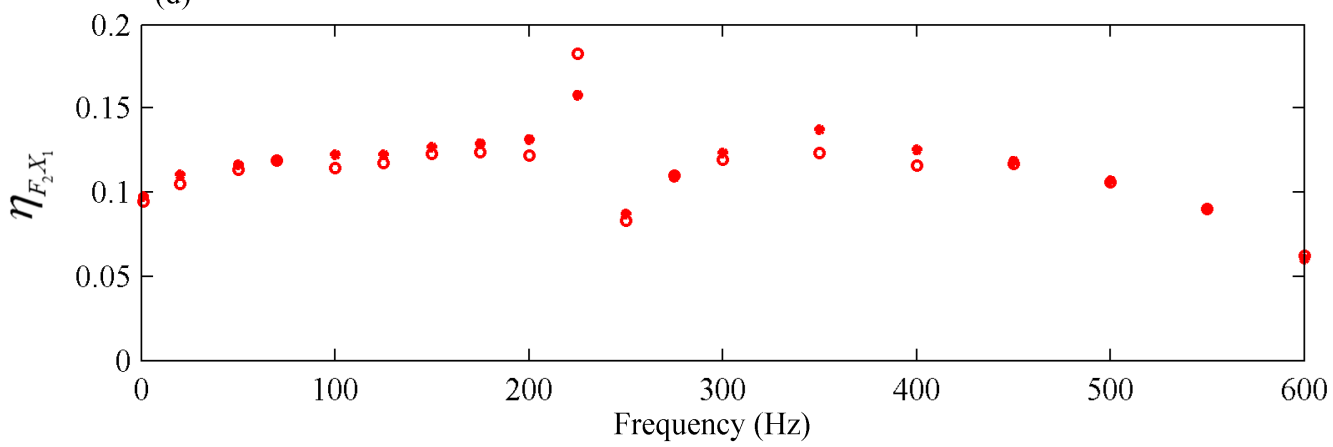

Figure 6: Measured cross-point dynamic stiffness showing magnitude (a, b) and loss factor (c, d). Key:

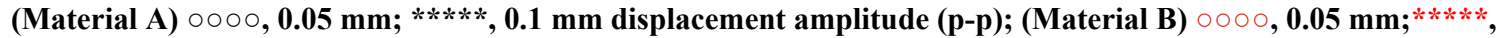
$0.05 \mathrm{~mm}$ displacement amplitude (p-p). 


\subsection{Modeling the Direct Identification Experiment Using a Finite Element Model}

The inverse identification experiment is constructed to identify the driving-point properties of the elastomeric joint; thus, a finite element model of the cross-point measurement of the direct experiment is used to establish a driving-point comparison. The direct uniaxial characterization experiments are used to determine the effective elastic modulus, loss factor, and density of the two elastomeric materials. A finite element model of the direct fixture is used to determine the material parameters of the joint elastic material. It is recognized that material nonlinearities are present; however, the assumption is made that at small displacements above say $50 \mathrm{~Hz}$, the elastomer can be approximated as a linear elastic material with structural (hysteretic) damping proportional to stiffness. The static stiffness can be found by choosing a value of the dynamic stiffness magnitude at low frequencies. From this stiffness value, $\tilde{k}_{\text {static }}$, the modulus of rigidity is found with the following equation $\tilde{G}=\left(\tilde{k}_{\text {static }} L\right) / A$, where $L$ is the length of the elastic material of the joint $(2.54$ $\mathrm{cm}$ ) and $A$ is the cross sectional area of a $2.54 \mathrm{~cm}$ diameter circle. The elastic modulus of the

structure can then be found by the relationship provided by the Poisson's ratio, $\tilde{E}=2 \tilde{G}(1+v)$, where the Poisson's ratio, $v$, is chosen as 0.49 for rubber. The dynamic stiffness and loss factor comparison between the experiment and FEA is displayed on Figure 7. The values found for the elastic moduli, loss factors, and densities of the joint elastic material are shown on Table 2. In order to now obtain simulated direct calculations comparable to the inverse driving point calculations, a simplified finite element model of a joint fixed on one end, as displayed on Figure $2 \mathrm{f}$, is used in the subsequent sections. 
Table 2: Identified material properties and parameters of the two materials.

\begin{tabular}{||c|c|c||}
\hline Material Property & Material A & Material B \\
\hline \hline Density, $\rho(\mathrm{kg} / \mathrm{m} 3)$ & 1300 & 1100 \\
Elastic Modulus, $\tilde{E}(\mathrm{MPa})$ & 15.38 & 8.38 \\
Loss Factor, $\eta$ & 0.06 & 0.12 \\
\hline
\end{tabular}

An example of the difference between a cross point measurement, $\tilde{k}_{F_{2} X_{1}}$, identified with the

direct fixture FEA and a driving point result, $\tilde{k}_{F_{1} X_{1}}$, using the fixed-free joint FEA of equation (1) are significant, as displayed on Figure 8. The cross point result shows a stiffness magnitude that increases with frequency due to an internal resonance of the joint; whereas, the driving point dynamic stiffness magnitude initially decreases before also increasing with frequency. It is apparent that a first natural frequency occurs at $150 \mathrm{~Hz}$ for material B and $200 \mathrm{~Hz}$ for material A. This same natural frequency can be seen to occur in the driving point loss factor results due to the loss factor dependence on the real part of the stiffness. Note that the metal cap of the elastomeric cylinder is lumped with the joint properties. 
(a)

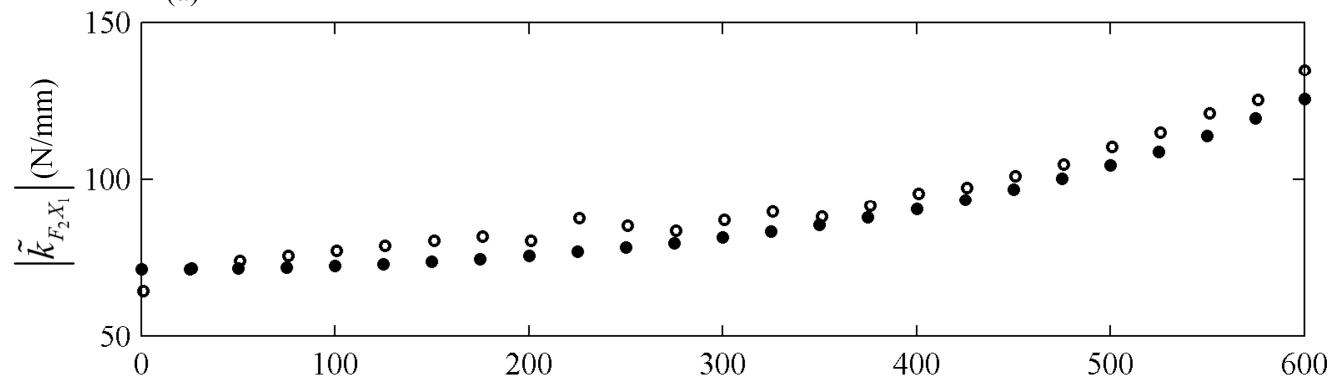

(b)

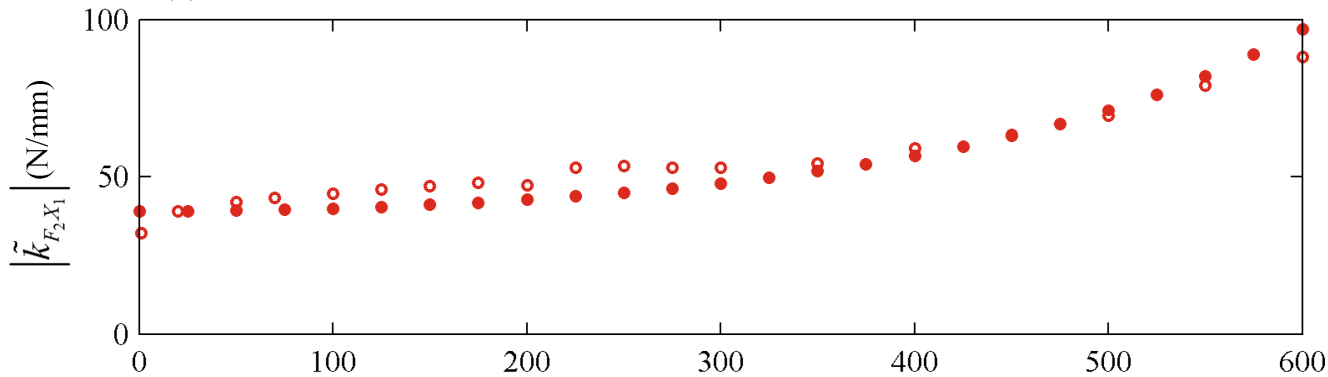

(c)

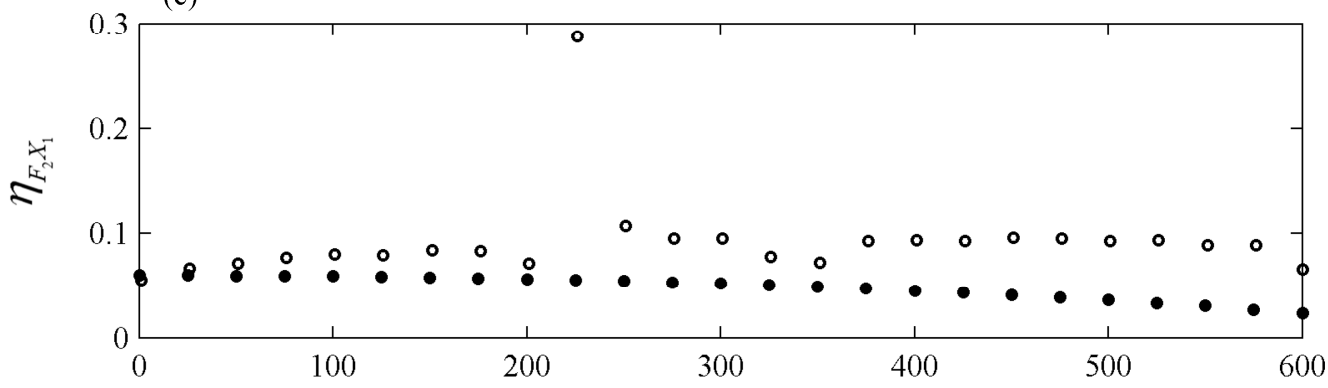

(d)

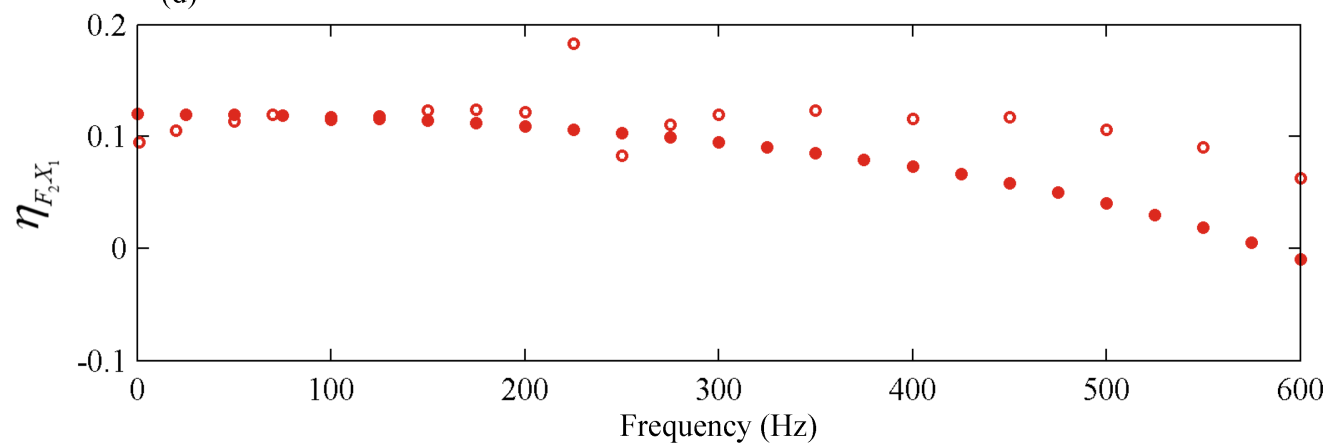

Figure 7: FEA approximation of experimental cross point dynamic stiffness magnitude (a, c) and loss factor (b,

d) results for Material A and Material B. Key: (Material A) $\bullet \bullet \bullet \bullet$, FEA; $০ \circ ০ \circ$, Experiment; (Material B) $\bullet \bullet \bullet$, FEA; $\diamond \circ \circ \diamond$, Experiment. 


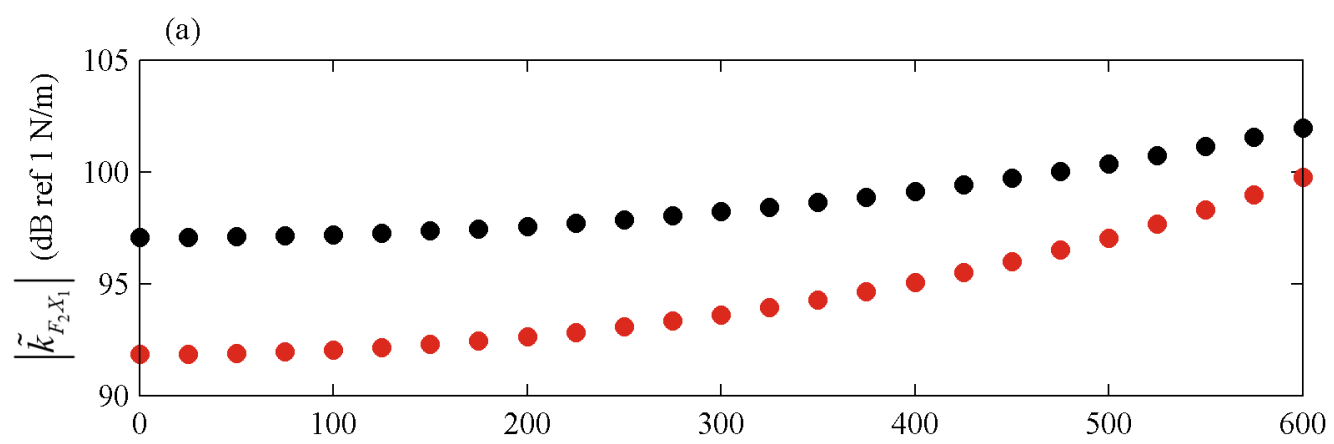

(b)

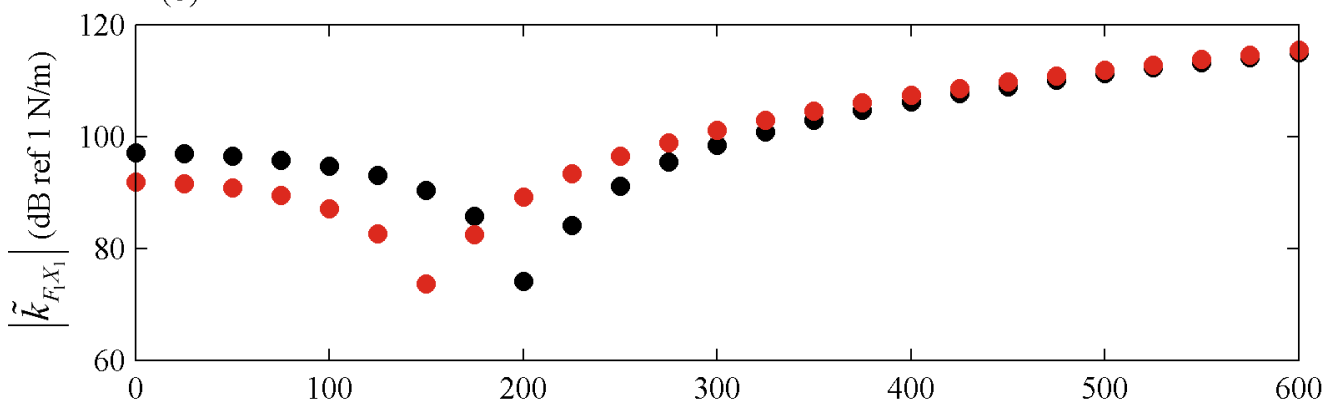

(c)

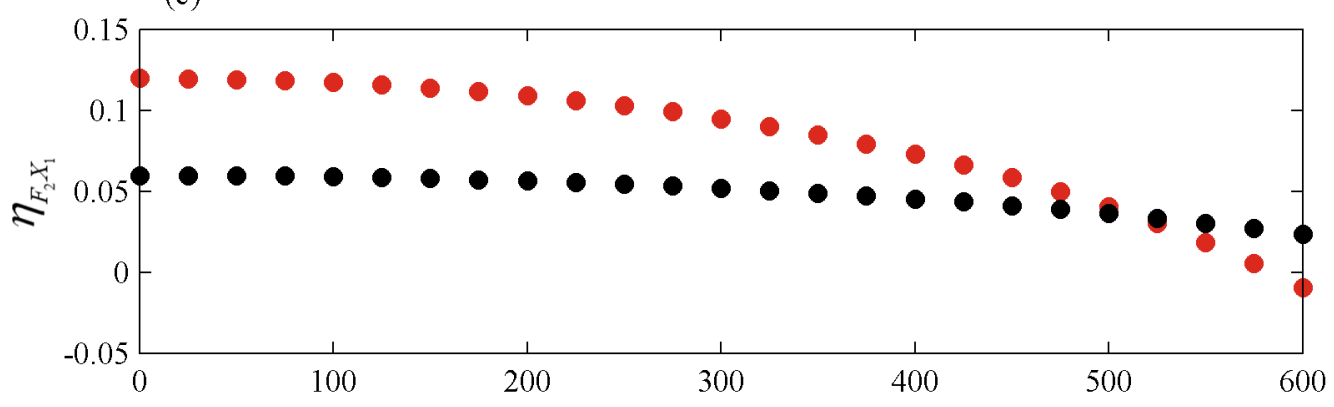

(d)

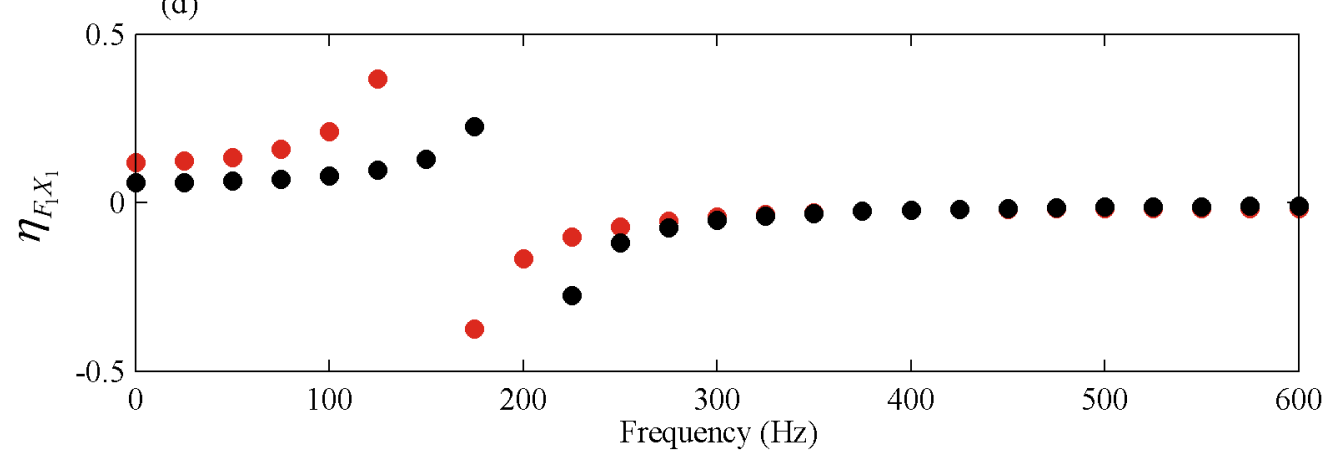

Figure 8: Identified results for cross point dynamic stiffness magnitude (a), driving point dynamic stiffness magnitude (b), cross point loss factor (c), and driving point loss factor (d) for the joint finite element simulation.

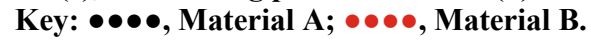




\section{Chapter 3: Inverse Method for Identification}

Hong et al.'s method $[6,7]$ requires three dynamic stiffness matrices to identify the unknown joint properties. The three dynamic stiffness matrices consist of the dynamic stiffness matrix of the constrained structure $\left(\boldsymbol{D}^{*}\right)$, unconstrained structure $\left(\boldsymbol{D}^{o}\right)$ and the unknown joint properties $\left(\boldsymbol{D}^{u}\right)$ as illustrated on Figure 9. To summarize the method, if one had the dynamic stiffness matrix of the constrained and unconstrained structure, the unknown joint properties could be found from the subtraction of the two matrices. In most applications, the constrained structure is not completely known. This requires the need to estimate unobserved frequency response functions [7]. Joint locations are typically unobserved coordinates due to obstructions and lack of experimental equipment; yet, these frequency response functions are required for estimation of the unknown joint properties in the identification formula. This leads to this method requiring two steps. First, one must use the estimation formula for unobserved frequency response functions. The second step is to use the identification formula for unknown joint properties. The next two sections formulate the derivation for these two equations. The mathematical formulation is then implemented into a Matlab script [9].

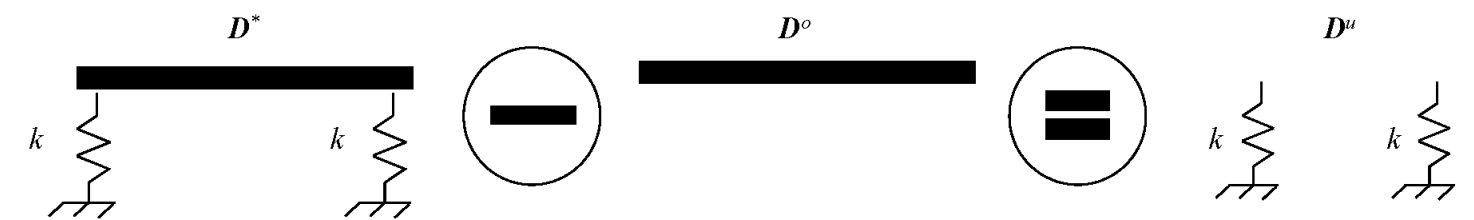

Figure 9: Simplified illustration of the identification method and the corresponding dynamic stiffness matrices where $k$ is a stiffness element. 


$$
\boldsymbol{D}^{*}-\boldsymbol{D}^{o}=\boldsymbol{D}^{u}
$$

\subsection{Derivation of Formulas}

\subsubsection{Estimation Formula for Unobserved Frequency Response Functions}

The dynamic stiffness matrix of a structural dynamic system is defined based on the physical domain matrices mass $(\boldsymbol{M})$, stiffness $(\boldsymbol{K})$, and viscous damping $(\boldsymbol{C})$ :

$$
\boldsymbol{D}(j \omega)=-\omega^{2} \boldsymbol{M}+j \omega \boldsymbol{C}+\boldsymbol{K} .
$$

The constrained structure is composed of the freely suspended structure as well as the joints written as

$$
\boldsymbol{D}^{*}(j \omega) \boldsymbol{q}(j \omega)=\left\{\boldsymbol{D}_{o}(j \omega)+\boldsymbol{D}_{j}^{*}(j \omega)\right\} \boldsymbol{q}(j \omega)=\boldsymbol{F}(j \omega),
$$

where $\boldsymbol{D}_{o}(j \omega)=-\omega^{2} \boldsymbol{M}_{o}+j \omega \boldsymbol{C}_{o}^{*}+\boldsymbol{K}_{o}^{*}$, and $\boldsymbol{D}_{j}^{*}(j \omega)=-\omega^{2} \boldsymbol{M}_{j}^{*}+j \omega \boldsymbol{C}_{j}^{*}+\boldsymbol{K}_{j}^{*}$, and the superscript “*”, and subscripts " $O$ " and " $j "$ represent the constrained, unconstrained and joint structures respectively. $\boldsymbol{q}$ represents the coordinate vector and $\boldsymbol{F}$ is the force vector. $\boldsymbol{M}_{o}, \boldsymbol{C}_{o}$ and $\boldsymbol{K}_{o}$ represent the mass, viscous damping and stiffness matrices of the unconstrained system and $\boldsymbol{M}_{j}, \boldsymbol{C}_{j}$ and $\boldsymbol{K}_{j}$ are the mass, viscous damping and stiffness matrices of the joints. The coordinate vectors are defined in terms of selection matrices designating specific coordinates on the constrained structure (Figure 10):

$$
\begin{aligned}
& \boldsymbol{q}_{n}^{(n \times 1)}=\boldsymbol{T}_{n}^{(n \times N)} \boldsymbol{q}^{(N \times 1)} \\
& \boldsymbol{q}_{\bar{n}}^{(\bar{n} \times 1)}=\boldsymbol{T}_{\bar{n}}^{(\bar{n} \times N)} \boldsymbol{q}^{(N \times 1)} \\
& \boldsymbol{q}_{m}^{(m \times 1)}=\boldsymbol{T}_{m}^{(m \times N)} \boldsymbol{q}^{(N \times 1)} \\
& \boldsymbol{q}_{\bar{m}}^{(\bar{m} \times 1)}=\boldsymbol{T}_{\bar{m}}^{(\bar{m} \times N)} \boldsymbol{q}^{(N \times 1)} \\
& \boldsymbol{q}_{e}^{(\mathrm{e} \times 1)}=\boldsymbol{T}_{e}^{(\mathrm{e} \times N)} \boldsymbol{q}^{(N \times 1)}
\end{aligned}
$$


where $n, \bar{n}, m, \bar{m}, e$ and $N$ designate the coordinates connected to joints, coordinates not connected to joints, observed coordinates, unobserved coordinates, excitation coordinates and total number of coordinates on the beam, respectively.

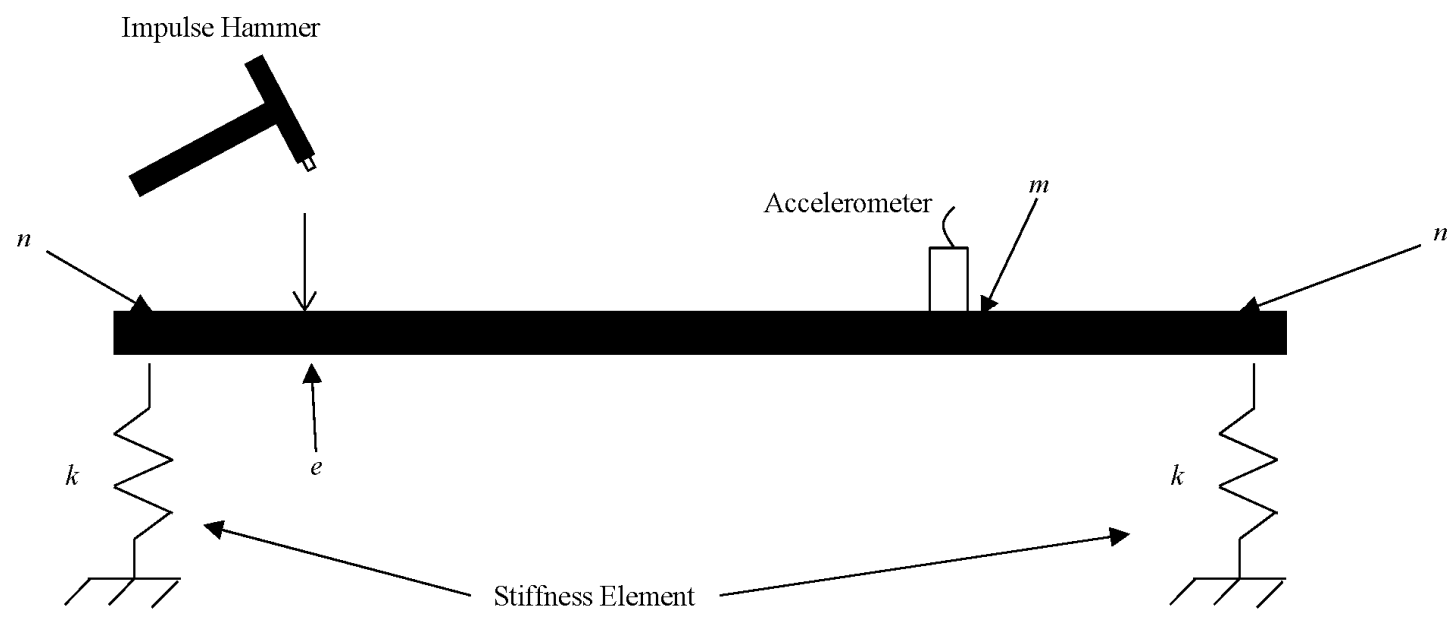

Figure 10: Illustration of important coordinates relevant to the identification method.

$\boldsymbol{T}$ is a selection matrix composed of 1 and 0 in order to isolate the coordinates of the attached subscript. The dynamic stiffness matrix of only joint coordinates is sparse except at the joint locations, so the joint dynamic stiffness matrix can be condensed to

$$
\boldsymbol{D}_{j}^{c}(j \omega)=\boldsymbol{T}_{n} \boldsymbol{D}_{j}^{*}(j \omega) \boldsymbol{T}_{n}^{t},
$$

where " $c$ " designates a condensed matrix of the order $n \times n$.

Often, when using this method, the constrained dynamic stiffness matrix is not known. This will require estimation of frequency response functions at the unobserved coordinates. In order to estimate the unobserved frequency response functions, consider an auxiliary model designated by superscript " $a$ ". The dynamic stiffness matrix for this model is 


$$
\boldsymbol{D}^{a}(j \omega)=\boldsymbol{D}_{o}(j \omega)+\boldsymbol{D}_{j}^{a}(j \omega)
$$

where

$$
\boldsymbol{D}_{j}^{a}(j \omega)=-\omega^{2} \boldsymbol{M}_{j}^{a}+j \omega \boldsymbol{C}_{j}^{a}+\boldsymbol{K}_{j}^{a}
$$

Notice that the only difference between the auxiliary and actual system is the joint dynamic stiffness matrix. The definition of frequency response matrix gives

$$
\boldsymbol{D}^{*}(j \omega) \boldsymbol{H}^{*}(j \omega)=\boldsymbol{I},
$$

where $\boldsymbol{H}^{*}$ is the dynamic compliance matrix of the constrained structure, and $\boldsymbol{I}$ is the identity matrix. Since the dynamic stiffness of the actual model and the auxiliary model are the same except at the joint coordinates, the following is true:

$$
\boldsymbol{T}_{\bar{n}} \boldsymbol{D}_{o}(j \omega)=\boldsymbol{T}_{\bar{n}} \boldsymbol{D}^{*}(j \omega)
$$

Substituting Equation (9) into Equation (10) and postmultiplying by $\boldsymbol{T}_{e}$, Equation (11) can be written as

$$
\boldsymbol{T}_{\bar{n}} \boldsymbol{D}_{o}(j \omega) \boldsymbol{H}^{*}(j \omega) \boldsymbol{T}_{e}^{t}=\boldsymbol{T}_{\bar{n}} \boldsymbol{T}_{e}^{t}
$$

This can then be arranged into unobserved and observed coordinates using selection matrices:

$$
\boldsymbol{T}_{n} \boldsymbol{D}_{o}(j \omega)\left[\begin{array}{ll}
\boldsymbol{T}_{m}^{t} & \boldsymbol{T}_{\bar{m}}^{t}
\end{array}\right]\left[\begin{array}{l}
\boldsymbol{T}_{m} \\
\boldsymbol{T}_{\bar{m}}
\end{array}\right] \boldsymbol{H}^{*}(j \omega) \boldsymbol{T}_{e}^{t}=\boldsymbol{T}_{n} \boldsymbol{T}_{e}^{t} .
$$

Equation (13) can be rewritten in the following form by using condensed dynamic stiffness matrices: 


$$
\left[\begin{array}{ll}
\boldsymbol{D}_{\bar{n} m}(j \omega) & \boldsymbol{D}_{\bar{n} \bar{m}}(j \omega)
\end{array}\right]\left[\begin{array}{l}
\boldsymbol{H}_{m e}^{*}(j \omega) \\
\boldsymbol{H}_{\bar{m} e}^{*}(j \omega)
\end{array}\right]=\boldsymbol{T}_{\bar{n}} \boldsymbol{T}_{e}^{t}
$$

where the condensed dynamic stiffness and compliance matrices are defined as $\boldsymbol{D}_{\bar{n} m}=\boldsymbol{T}_{\bar{n}} \boldsymbol{D}_{o} \boldsymbol{T}_{m}^{t}$, $\boldsymbol{D}_{\bar{n} \bar{m}}=\boldsymbol{T}_{\bar{n}} \boldsymbol{D}_{o} \boldsymbol{T}_{\bar{m}}^{t}, \quad \boldsymbol{H}_{m e}^{*}=\boldsymbol{T}_{m} \boldsymbol{H}^{*} \boldsymbol{T}_{e}^{t}, \boldsymbol{H}_{\bar{m} e}^{*}=\boldsymbol{T}_{\bar{m}} \boldsymbol{H}^{*} \boldsymbol{T}_{e}^{t}$. Equation (13) can be arranged to solve for the following estimated compliance frequency response functions:

$$
\boldsymbol{H}_{\bar{m} e}^{*}(j \omega)=\left[\boldsymbol{D}_{\bar{n} \bar{m}}(j \omega)\right]^{L}\left[\boldsymbol{T}_{\bar{n}} \boldsymbol{T}_{e}^{t}-\boldsymbol{D}_{\bar{n} m}(j \omega) \boldsymbol{H}_{m e}^{*}(j \omega)\right],
$$

where the superscript " $L$ " designates the generalized left inverse matrix. There are a necessary number of observed frequency response functions for accurate estimation of frequency response functions at unobserved coordinates. This number needs to be at least equal to the number of unknown joint properties being identified.

\subsubsection{Identification Formula for Unknown Joint Properties}

The second step in the inverse identification method, developed by Hong et al. [7], consists of using the identification formula for unknown joint properties. This derivation begins with first identifying $\boldsymbol{D}_{u}$ which is written as

$$
\boldsymbol{D}_{u}(j \omega)=\boldsymbol{D}^{*}(j \omega)-\boldsymbol{D}^{a}(j \omega)=\boldsymbol{D}_{j}^{*}(j \omega)-\boldsymbol{D}_{j}^{a}(j \omega),
$$

where the subscript " $u$ " denotes the unknown joint coordinates that are found through the inverse identification method. Using the definition of dynamic stiffness and by premultiplying by $\boldsymbol{H}^{a}$ and postmultiplying by $\boldsymbol{H}^{*}$ Equation (16) can be given as

$$
\boldsymbol{H}^{a}(j \omega)-\boldsymbol{H}^{*}(j \omega)=\boldsymbol{H}^{a}(j \omega) \boldsymbol{D}_{u}(j \omega) \boldsymbol{H}^{*}(j \omega) .
$$

From Equations (6), (7) and (8), $\boldsymbol{D}_{u}$ can be condensed to 


$$
\boldsymbol{D}_{u}^{c}(j \omega)=\boldsymbol{T}_{n}(j \omega) \boldsymbol{D}_{u}^{*}(j \omega) \boldsymbol{T}_{n}(j \omega)
$$

Substituting equation (17) into equation (16), premultiplication of $\boldsymbol{T}_{m}$ and postmultiplcation of $\boldsymbol{T}_{e}^{t}$ to both sides gives

$$
\boldsymbol{H}_{m e}^{a}-\boldsymbol{H}_{m e}^{*}=\boldsymbol{H}_{m n}^{a}(j \omega) \boldsymbol{D}_{u}^{c}(j \omega) \boldsymbol{H}_{n e}^{*}(j \omega),
$$

which can be rearranged to solve for $\boldsymbol{D}_{u}^{c}$ yielding the identification formula for unknown joint properties:

$$
\boldsymbol{D}_{u}^{c}(j \omega)=\left[\boldsymbol{H}_{m n}^{a}(j \omega)\right]^{L}\left[\boldsymbol{H}_{m e}^{a}(j \omega)-\boldsymbol{H}_{m e}^{*}(j \omega)\right]\left[\boldsymbol{H}_{n e}^{*}(j \omega)\right]^{R},
$$

where the superscript " $R$ " is the generalized right hand inverse.

\subsection{Inverse Beam Experiment}

Figure 11a shows the constrained tractable beam experiment (constrained structure) which consists of the aluminum beam connected to ground through the joint. A finite element model of a three feet long 0.5 in $\times 0.5$ in aluminum cross sectioned beam composed of 19 nodes is used for the freely suspended unconstrained structure (Figure $11 \mathrm{~b}, \mathrm{c}$ ) in the inverse identification method. Timoshenko mass and stiffness beam elements [10] are used and assume rotational and transverse motion of the beam. The stiffness element of a Timoshenko beam is given by

$$
\boldsymbol{K}_{\varepsilon}=\frac{E I}{(1+\phi) L^{3}}\left[\begin{array}{cccc}
12 & 6 L & -12 & 6 L \\
& (4+\phi) L^{2} & -6 & (2-\phi) L^{2} \\
& & 12 & -6 L \\
\text { symmetric } & & & (4+\phi) L^{2}
\end{array}\right],
$$


where $E$ is the elastic modulus, $I$ is the area moment of inertia, $L$ is the element length ( 2 in), and $\phi$ is the ratio of beam bending stiffness to shear stiffness. The area moment of inertia of a square cross-section is

$$
I=\frac{h^{4}}{12}
$$

where $h$ is the length of a cross section edge and $\phi$ is defined by

$$
\phi=\frac{12}{L^{2}}\left(\frac{E I}{\lambda G A}\right)(1+v)
$$

where $v$ is Poisson's ratio chosen as .33 for aluminum, $G$ is the modulus of rigidity, $A$ is the cross sectional area of the beam and $\lambda$ is the shear correction factor for the rectangular beam taken to be

$$
\lambda=\frac{10(1+v)}{12+11 v} .
$$

Damping for the unconstrained structure is modeled as structural damping. The consistent mass matrix for Timoshenko beam elements is composed of two parts:

$$
\boldsymbol{M}_{e}=\boldsymbol{M}_{\rho A}+\boldsymbol{M}_{\rho I}
$$

where $\boldsymbol{M}_{\rho A}$ is associated with the translational inertia given by

$$
\boldsymbol{M}_{\rho A}=\frac{\rho A L}{210(1+\phi)^{2}}\left[\begin{array}{ccccc}
70 \phi^{2}+147 \phi+78 & \left(35 \phi^{2}+77 \phi+44\right) \frac{L}{4} & \left(35 \phi^{2}+63 \phi+27\right) & \ldots \\
& \left(7 \phi^{2}+14 \phi+8\right) \frac{L^{2}}{4} & \left(35 \phi^{2}+63 \phi+26\right) \frac{L}{4} & \ldots \\
& & 70 \phi^{2}+147 \phi+78 & \ldots \\
\text { symmetric } & & \ldots
\end{array}\right.
$$




$$
\left.\begin{array}{cc}
\ldots & -\left(35 \phi^{2}+63 \phi+26\right) \frac{L}{4} \\
\ldots & -\left(7 \phi^{2}+14 \phi+6\right) \frac{L^{2}}{4} \\
\ldots & -6 L \\
\ldots & \left(7 \phi^{2}+14 \phi+6\right) \frac{L^{2}}{4}
\end{array}\right]
$$

And $\boldsymbol{M}_{\rho I}$ is associated with the rotatory inertia given by

$$
\boldsymbol{M}_{\rho I}=\frac{\rho I}{30(1+\phi)^{2} L}\left[\begin{array}{cccc}
30 & -(15 \phi-3) L & -36 & -(35-3) L \\
& \left(10 \phi^{2}+5 \phi+4\right) L^{2} & (15-3) L & \left(5 \phi^{2}-5 \phi+1\right) L^{2} \\
& & 70 \phi^{2}+147 \phi+78 & (15 \phi-3) L \\
\text { symmetric } & & & \left(10 \phi^{2}+5 \phi+4\right) L^{2}
\end{array}\right]
$$

The global mass matrix is created from 18 of the element mass matrices. At common nodes, the element indices become additive. Similarly, the global stiffness matrix can be created [11].

The beam material properties are found by using a computational model of the free-free beam and comparing driving point compliance frequency response functions of the experimental beam with free bounday conditions for a frequency range of $0-1000 \mathrm{~Hz}$. The elastic modulus, density and loss factor of the aluminum beam are found to be $69 \mathrm{GN} / \mathrm{m}^{2}, 2700 \mathrm{~kg} / \mathrm{m}^{3}$ and .006 respectively.

By incorporating the geometric stiffness matrix developed by Kosmatka [12], preloaded structures can potentially be analyzed; however, this is left for future research. This geometric stiffness matrix, $\boldsymbol{K}_{g}$, for one element with transverse and rotational degrees of freedom is shown in the following equation:

$$
\boldsymbol{K}_{g}=\frac{1}{30 \mathrm{~L}(1+\phi)^{2}}\left[\begin{array}{cccc}
\left(36+60 \phi+30 \phi^{2}\right) & 3 L & -\left(36+60 \phi+30 \phi^{2}\right) & 3 L \\
& \left(4+5 \phi+2.5 \phi^{2}\right) L^{2} & -3 L & -\left(1+5 \phi+2.5 \phi^{2}\right) L^{2} \\
\text { symmetric } & & \left(36+60 \phi+30 \phi^{2}\right. & -3 L \\
& & & \left(4+5 \phi+2.5 \phi^{2}\right) L^{2}
\end{array}\right]
$$


(a)

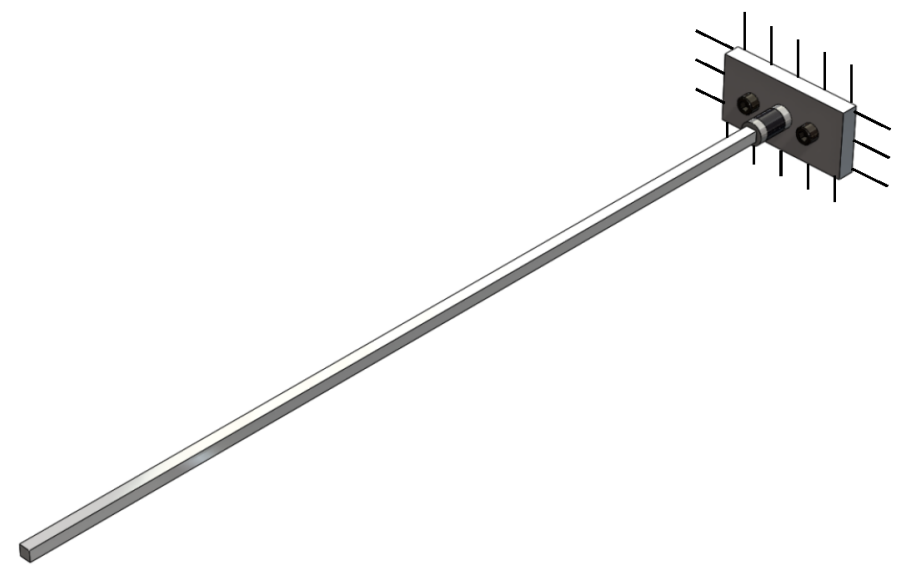

(b)

(c)

Element 1

Element 18

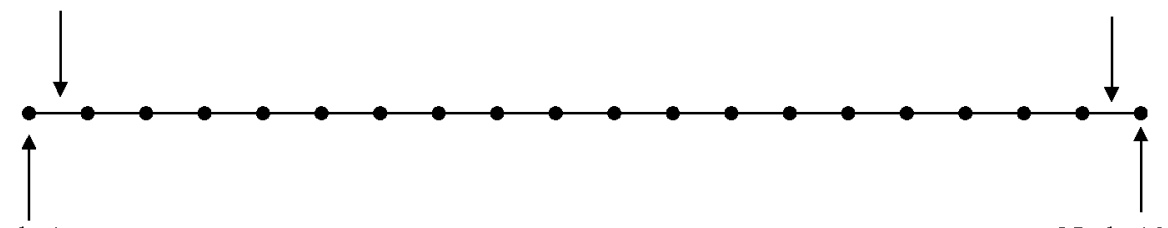

Node 1

Node 19

Figure 11: Inverse beam models. (a) Conceptual model of constrained structure, (b) conceptual model of unconstrained structure and (c) finite element model of unconstrained structure. 


\section{Chapter 4: Application of the Inverse Method}

\subsection{Inverse Method Computational Study}

The inverse method is now applied to the constrained and unconstrained structures of Figure 11. The equations needed to apply the inverse identification method are Equations (14) and (19). These two equations consist of the estimation formula for unobserved compliance frequency response functions and the identification formula for unknown joint properties, respectively. These equations take into account frequency response functions from both the unconstrained structure as well as the constrained structure to identify joint properties. Observed frequency response functions, $\boldsymbol{H}_{m e}^{*}$. are found from the constrained structure and used to find the estimated frequency response functions at unobserved locations, $\boldsymbol{H}_{\bar{m} e}^{*}$. The unobserved compliance frequency response functions corresponding to the joint locations, $\boldsymbol{H}_{n e}^{*}$, are now used in the identification formula to find the joint properties. The number of observed and excited locations must at least equal the number of unknown joint degrees of freedom for a unique solution. In this model, there is a rotational and translational joint degree of freedom that must be found, so at a minimum, two excitations and two observed locations are required.

Equations (14) and (19) of the inverse method include three pseudo inverses of dynamic stiffness and compliance matrices. It is important to check the conditioning of these matrices in order to eliminate sources of error at singular values. For matrix $\boldsymbol{Q}$, the condition number is defined as the ratio of the maximum singular value to the minimum singular value at a given frequency [13]. 


$$
\kappa(\boldsymbol{Q})=\frac{\sigma_{\max }(\boldsymbol{Q})}{\sigma_{\min }(\boldsymbol{Q})}
$$

where $\sigma_{\max }(\boldsymbol{Q})$ and $\sigma_{\min }(\boldsymbol{Q})$ are the maximum and minimum singular values of matrix $\boldsymbol{Q}$. For material A, the three matrices that conditioning is checked for are $\boldsymbol{D}_{\bar{n} \bar{m}}^{o}, \boldsymbol{H}_{n e}^{*}$, and $\boldsymbol{H}_{m n}^{o}$. The effect of conditioning is demonstrated with two variations of the same finite element simulation of the constrained structure and unconstrained structures of Figure 11. Table 3, model 1 and model 2, displays the coordinates on the constrained structure used to illustrate the importance of matrix conditioning. There is a translational and rotational coordinate for each node. Only translational coordinates are used for observed and excited coordinates. The conditioning of model 1 and model 2 are very different as illustrated on Figure 12. Model 1 and model 2 have different singular values for all three matrices. It is noticed that choosing the location of observed and excited locations is important in determining at what frequency these singular values occur. Also, it can be noticed that adding more observed and excited locations does not always necessarily decrease the value of the condition number at certain frequencies; however, in all cases analyzed, increasing the number of observed and excited locations improves the identified results. Depending on the system, the condition number of one of the three matrices may be more important in causing sources of error than the others. For this simulation, the condition number of $\boldsymbol{D}_{\bar{n} \bar{m}}^{o}$ proves to be critical in creating a source of error for model 1 as shown on Figure 13. 
(a)

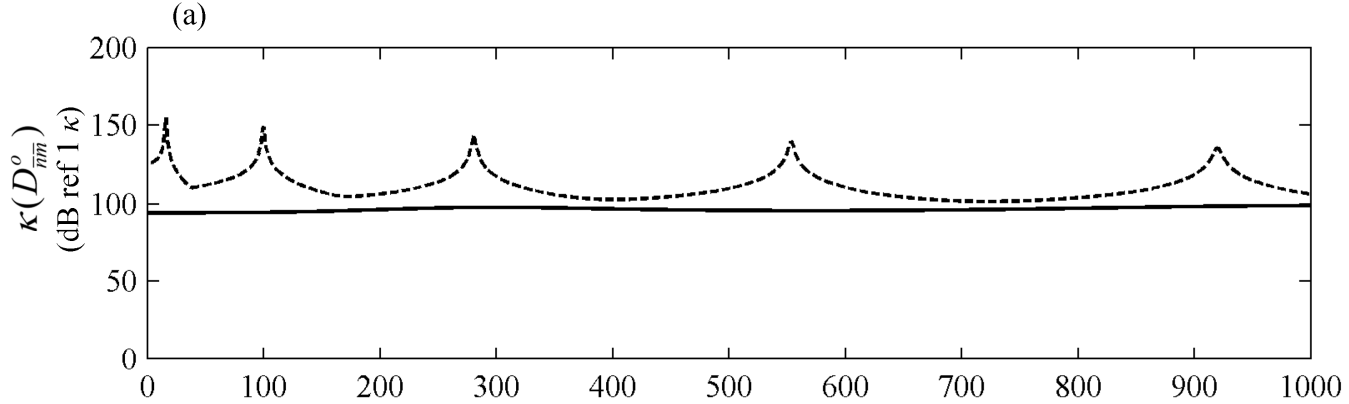

(b)
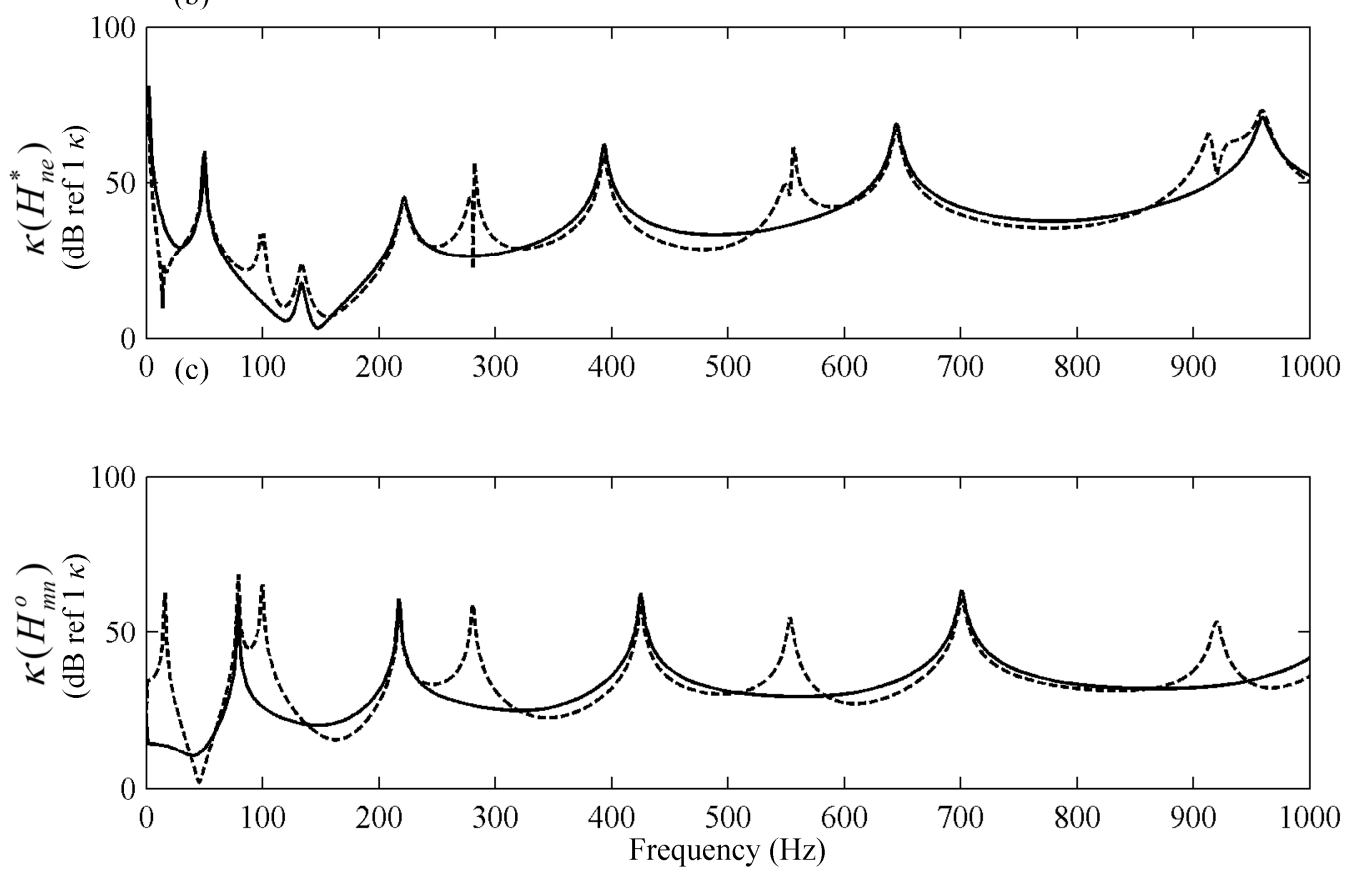

Figure 12: Conditioning of $\boldsymbol{D}_{\bar{n} \bar{m}}^{o}(\mathrm{a}), \boldsymbol{H}_{n e}^{*}(\mathrm{~b})$, and $\boldsymbol{H}_{m n}^{o}$ (c) for Models 1 and Model 2. Key: - - - -, Model 1; Model 2.

Figure 13 shows that at the location of singular values regarding $\boldsymbol{D}_{\bar{n} \bar{m}}^{o}$, significant error of the identifed translational stiffness magnitude and damping of the joints occurs. Since this is the case, an alternative model is prefered. It is noticed that with more observed and excited locations, error due to conditioning can be minimized. A final observation is that at increasing frequencies the error due to conditioning has more effect on the identified results. Identified results for model 
2, displayed on Figure 14, shows significantly less error at singular values of $\boldsymbol{D}_{\bar{n} \bar{m}}^{o}$. This model is used for the remainder of this chapter.

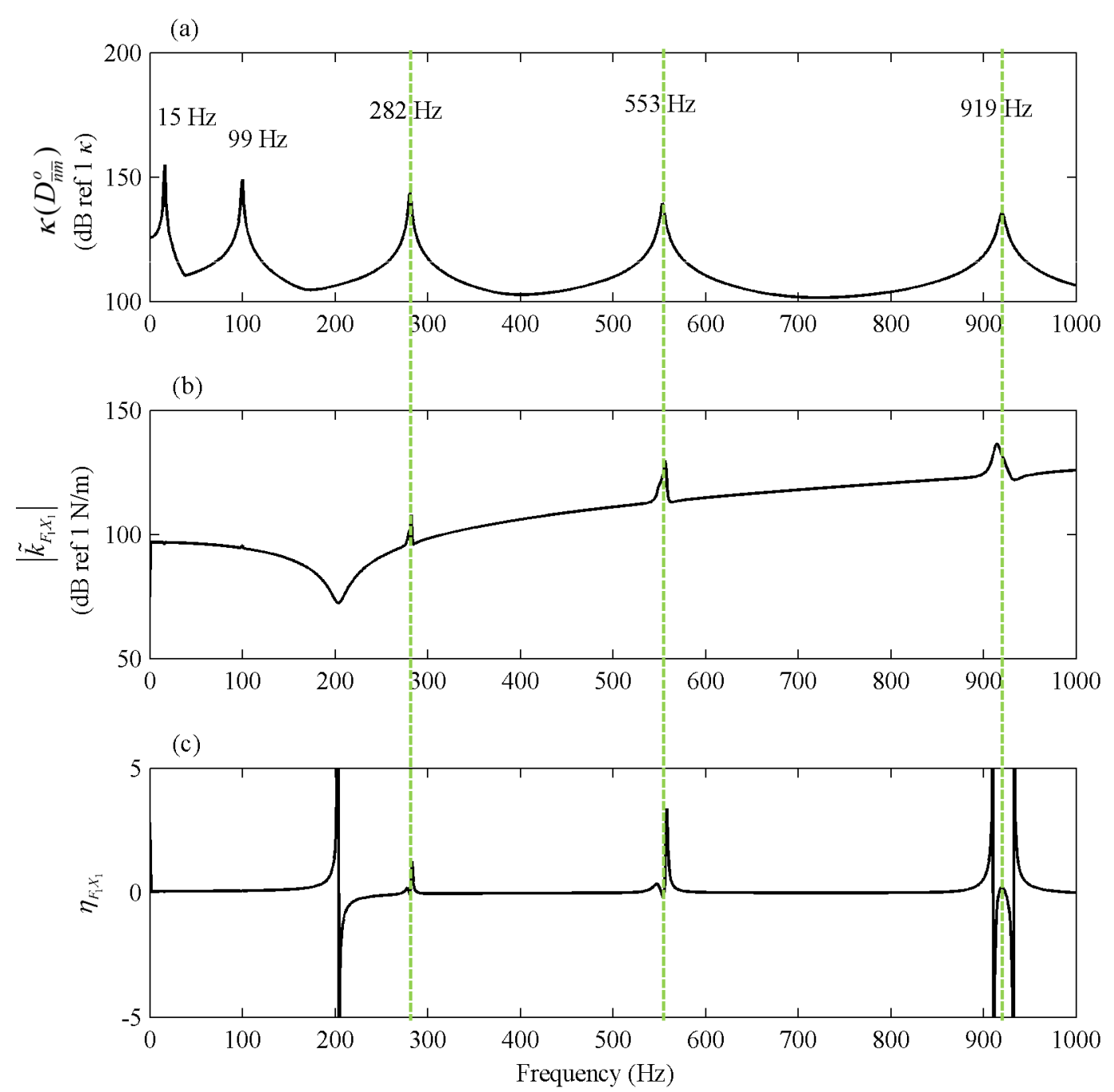

Figure 13: FEA identified translational dynamic stiffness magnitude (b) and loss factor (c) for Model 1 displaying effect of conditioning of $D_{\bar{n} \bar{m}}^{\circ}$ (a). 
(a)

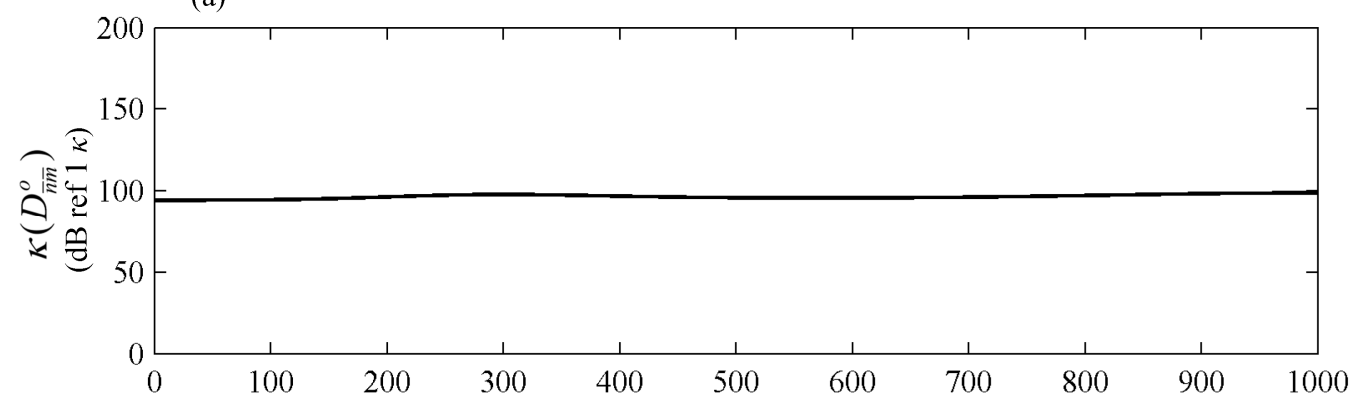

(b)

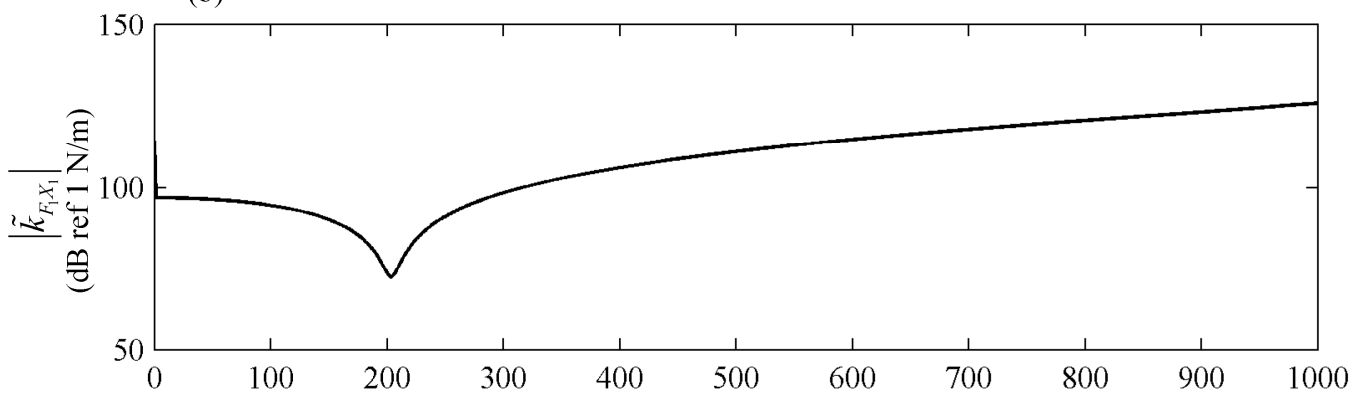

(c)

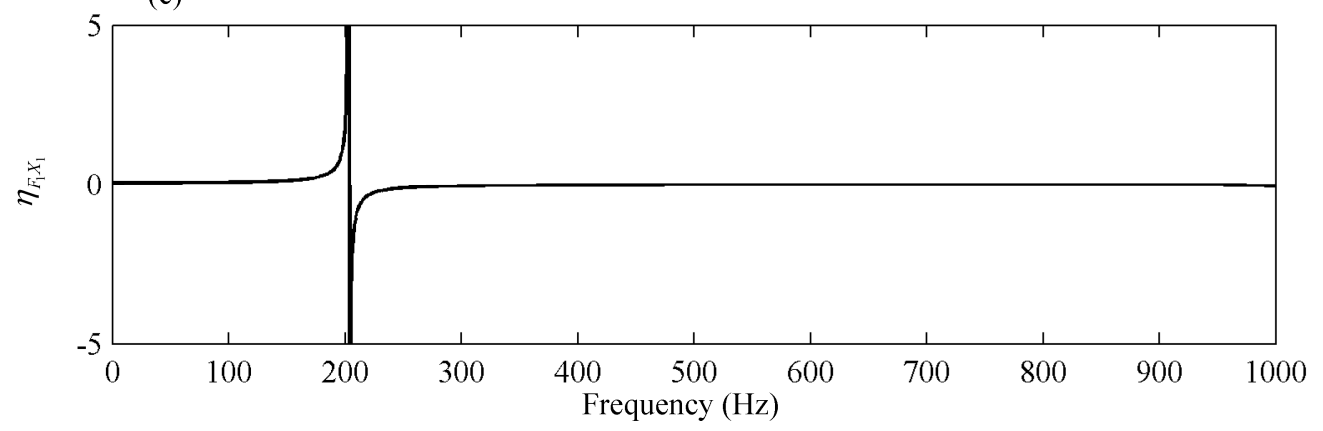

Figure 14: FEA identified translational dynamic stiffness magnitude (b) and loss factor (c) for Model 2 displaying effect of conditioning of $D_{\bar{n} \bar{m}}^{o}$ (a).

Table 3: Models used for inverse computational studies and experiment

\begin{tabular}{||c||c||c|c||}
\hline \multicolumn{4}{|c||}{ Coordinates for Inverse Models } \\
\hline \hline Model \# & Joints & Observed (Trans.) & Excited (Trans.) \\
\hline \hline Model 1 & 1 (Trans.), 1 (Rot.) & 2,4 & 2,4 \\
Model 2 & 1 (Trans.), 1 (Rot.) & $2,4,11,14,17$ & $2,4,11,14,17$ \\
Model 3 & 1 (Trans.), 1 (Rot.) & $2,3,4,5,11,14,17$ & $2,3,4,5,11,14,17$ \\
\hline
\end{tabular}




\subsection{Method Verification of the Identification Method}

Now that the tractable model for the inverse method has been designed and sufficient knowledge of conditioning has been discovered, it is important to verify the inverse results with that of the direct results. First, the direct identified stiffness properties have to be extended to 1000 $\mathrm{Hz}$, and the frequency resolution is now set to $1 \mathrm{~Hz}$ to match the frequency resolution of the inverse results. The identified stiffness and loss factor for material A, displayed on Figure 15, show good agreement for translational and coupling terms for the entire frequency range. Rotational terms also show good agreement but error occurs near the first mode.

(a)

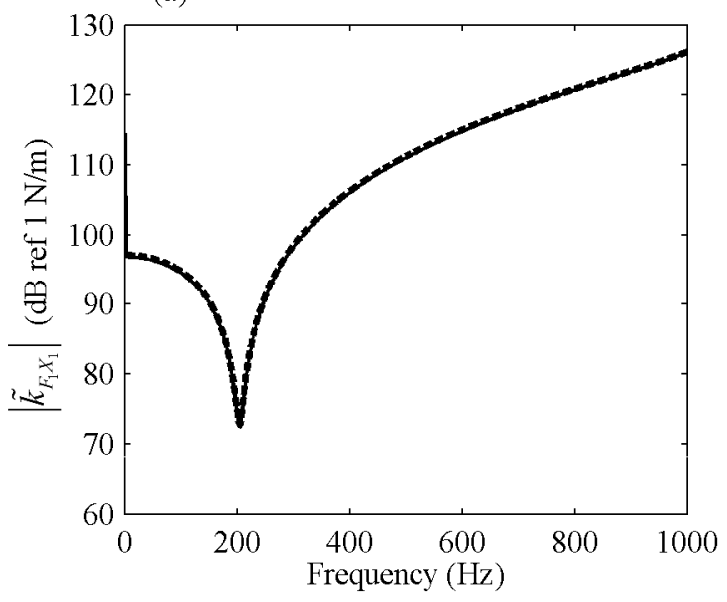

(b)

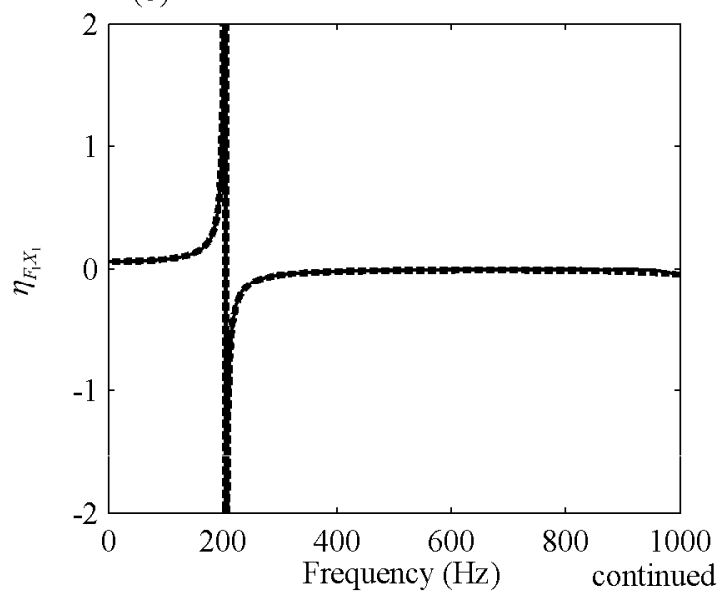

Figure 15: Verification of inverse stiffness magnitude and loss factor for Material A showing (a, b) Translational, (c, d) coupling, and (e, f) rotational stiffness components: Key: - - , Inverse; - . - -, Direct. 
(c)

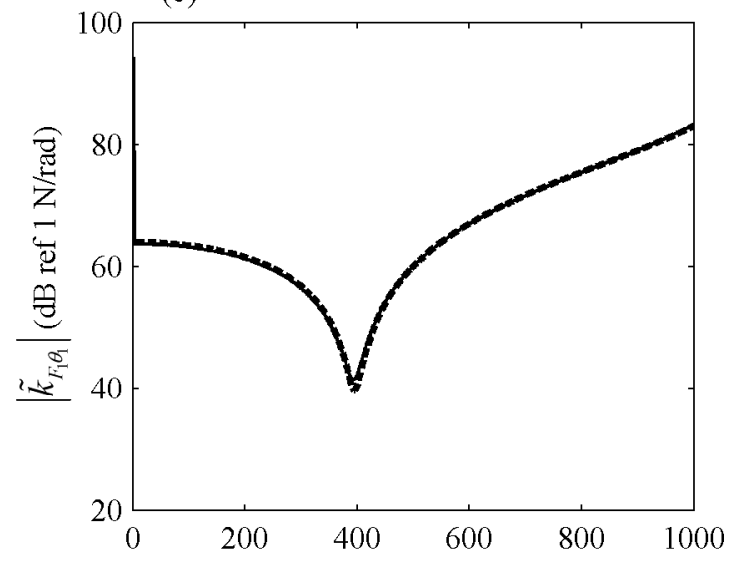

(e)

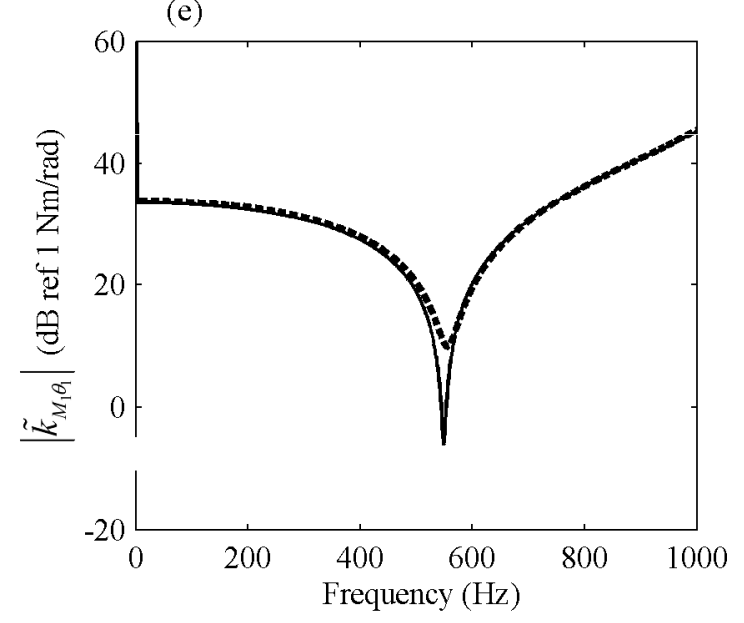

(d)

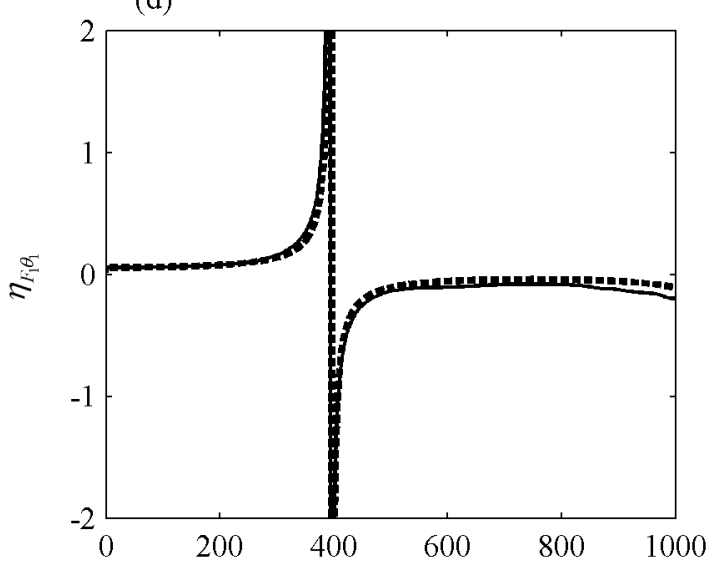

(f)

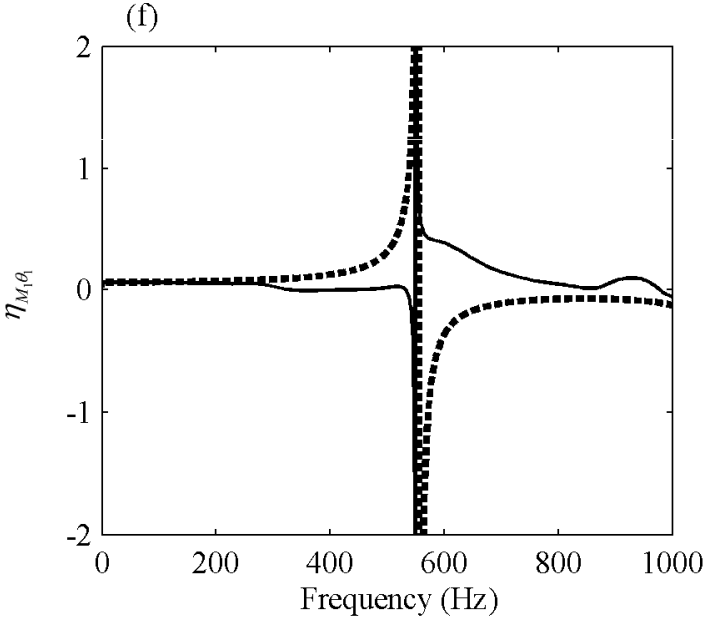

\subsection{Modeling Error}

Since analytical and experimental information are both utilized, the sensitivity to analytical modeling errors are examined in the context of the beam experiment. The finite element model of the inverse identification method is used to determine how modeling errors due to damping, density and elastic modulus could affect the identified joint properties. In experiments conducted by Colakoglu et al. [14], aluminum loss factor for a beam with free-free boundary conditions varied from $0.002-0.01$. Elastic modulus and density can often vary from one aluminum beam to the next. Aluminum elastic modulus and density are published as $69 \mathrm{GPa}$ and $2700 \mathrm{~kg} / \mathrm{m}^{3}$, respectively. It is 
important to see whether variance in these material properties can affect the identified results. This is accomplished by varying the damping, density and elastic modulus of the computational aluminum beam structure $\left(\eta_{o}, \rho_{o}\right.$, and $\left.E_{o}\right)$ in comparison to the constrained structure's aluminum beam loss factor, density and elastic modulus $\left(\eta_{*}, \rho_{*}\right.$, and $\left.E_{*}\right)$. The material properties are varied based on the following: $\eta_{o}=\left[\begin{array}{llllll}1 / 3 & 2 / 3 & 1 & 4 / 3 & 5 / 4\end{array}\right] \eta_{*}, \rho_{o}=\left[\begin{array}{lllll}0.98 & 0.99 & 1.00 & 1.01 & 1.02\end{array}\right] \rho_{*}$ and $E_{o}=\left[\begin{array}{lllll}0.98 & 0.99 & 1.00 & 1.01 & 1.02\end{array}\right] E_{*}$, where $\eta_{*}$ is chosen to equal $0.006, \rho_{*}$ is chosen as $2700 \mathrm{~kg} / \mathrm{m}^{3}$ and $E_{*}$ is chosen as $69 \mathrm{GPa}$. The translational, coupling and rotational terms associated with the following equations from the joint stiffness matrix of Equation (1) are compared to understand the effect of modeling error, where the translational stiffness term is $\tilde{k}_{F_{1} X_{1}} \cdot \tilde{k}_{F_{1} \theta_{1}}$ and $\tilde{k}_{M_{1} X_{1}}$ are the coupling terms and for the subject finite element model, $\tilde{k}_{F_{1} \theta_{1}}=\tilde{k}_{M_{1} X_{1}}$. The rotational term that is compared is $\tilde{k}_{M_{1} \theta_{1}}$. The analysis is completed for material A. Using model 2 of Table 3, Figures 16-18 show the identified joint properties and the stiffness magnitude and loss factor are affected by such modeling. It is noticed that rotational terms are the most sensitive to each of these modeling errors. Altering density and elastic modulus by $1 \%$ has detrimental effects on the accuracy of the inverse method as shown on Figure 17 and Figure 18. This newly gained information is important to consider when taking experimental measurements. For this experiment, the translational stiffness properties less sensitive to modeling errors than that of coupling and rotational; however, the coupling and rotational terms present further challenges. 
(a)

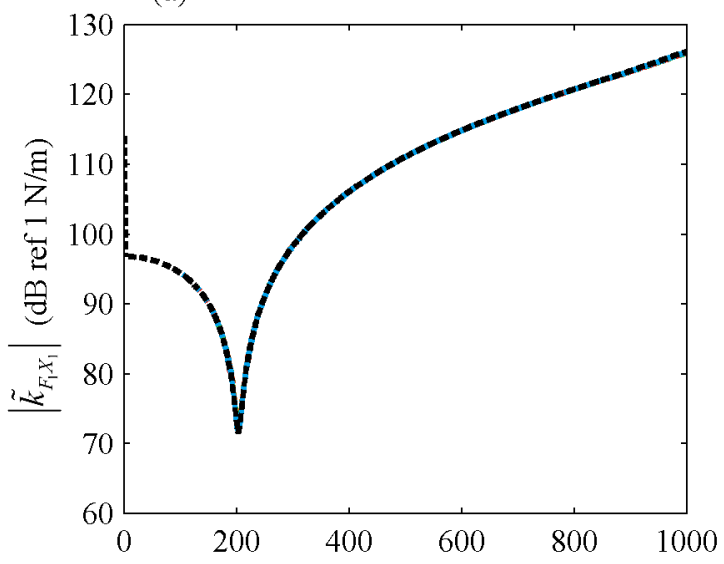

(c)

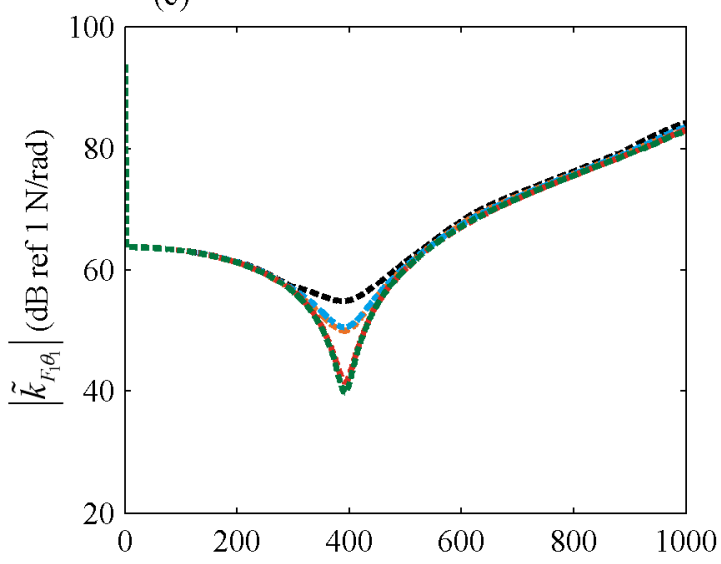

(e)

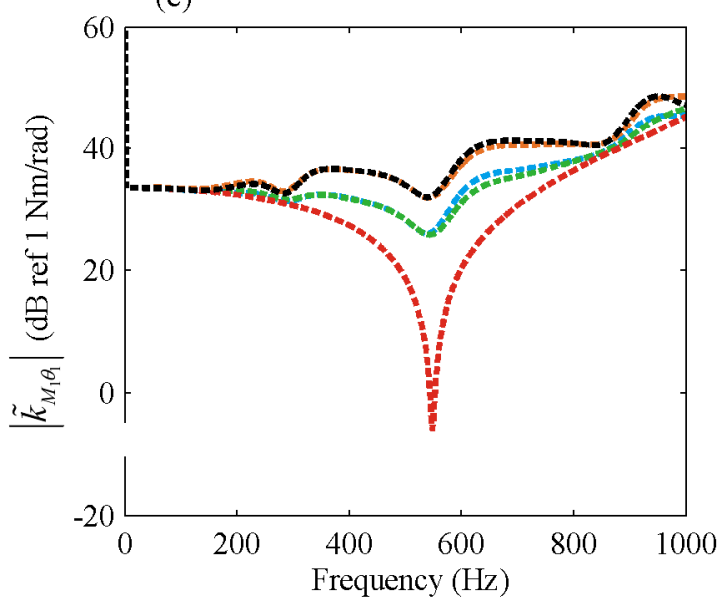

(b)

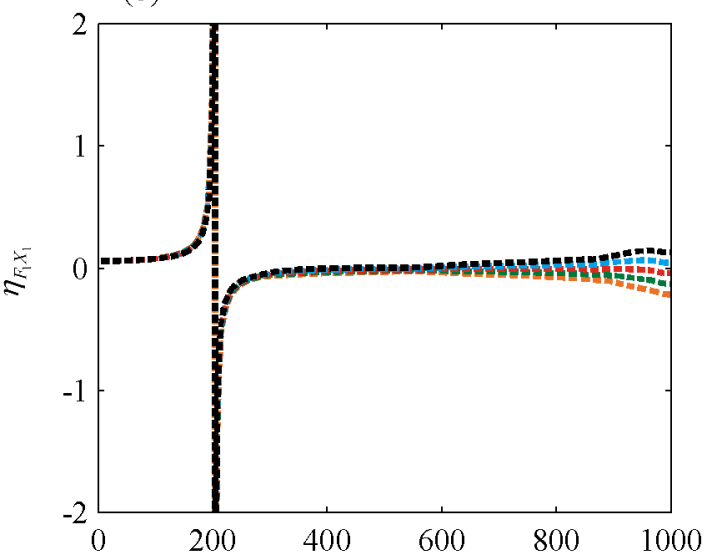

(d)
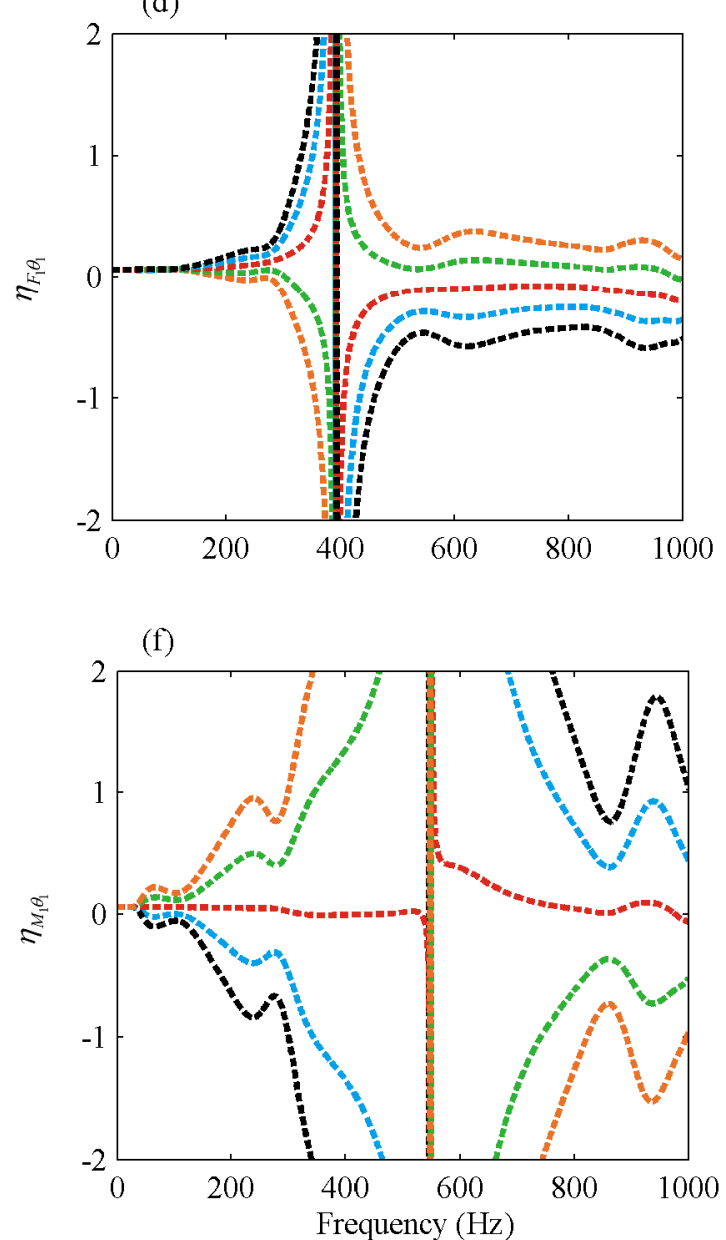

Figure 16: Inverse stiffness magnitude and loss factor for varied elastic modulus of unconstrained structure. (a, b) Translational, (c, d) coupling, and (e, f) rotational components: Key: $===, \eta_{o}=\eta^{*} / 3 ;==-=, \eta_{o}=2 \eta^{*} / 3 ;=-$ $=-, \eta_{o}=\eta * ;=-=, \eta_{o}=4 \eta^{*} / 3 ; \cdots,-\cdot \eta_{o}=5 \eta * / 3$. 
(a)

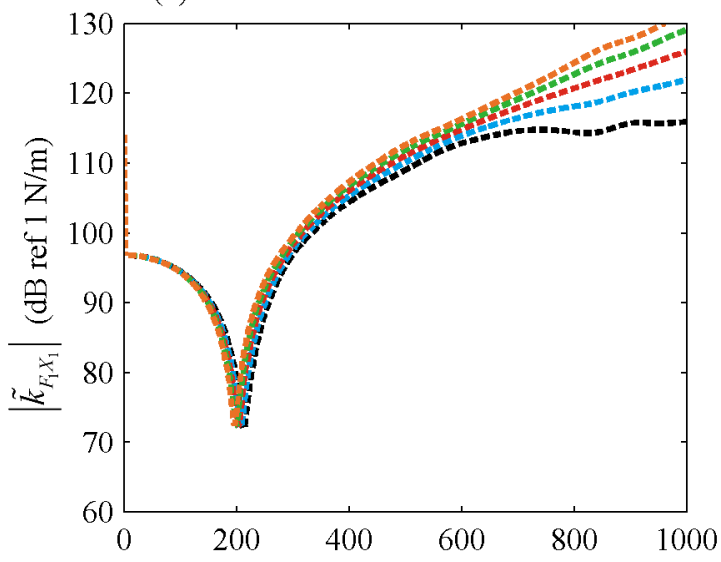

(c)

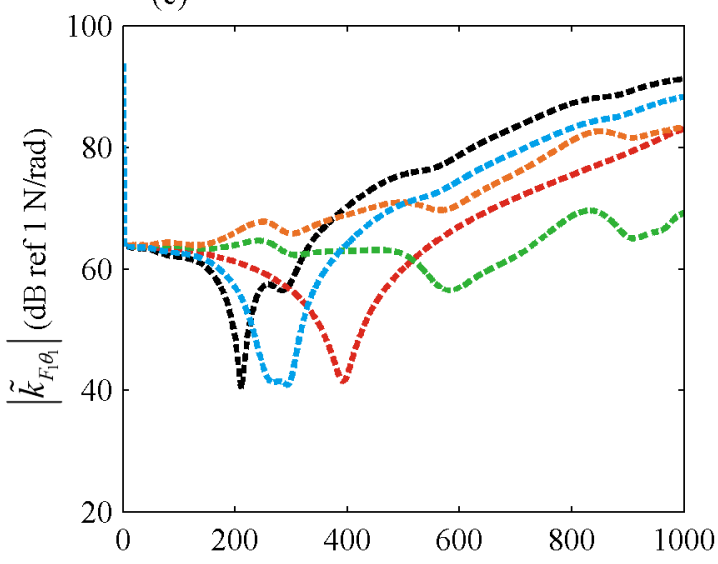

(e)

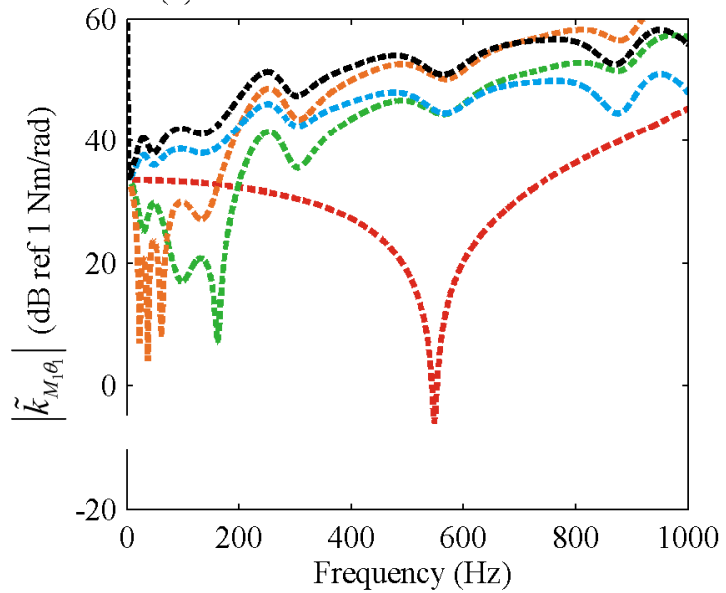

(b)

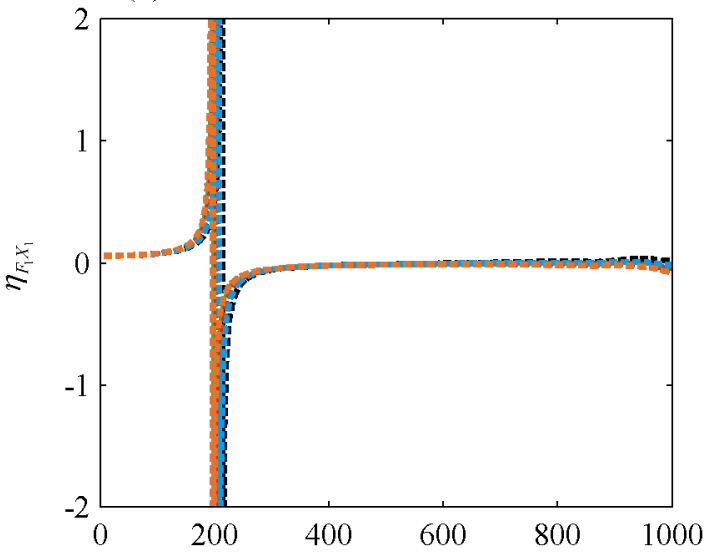

(d)

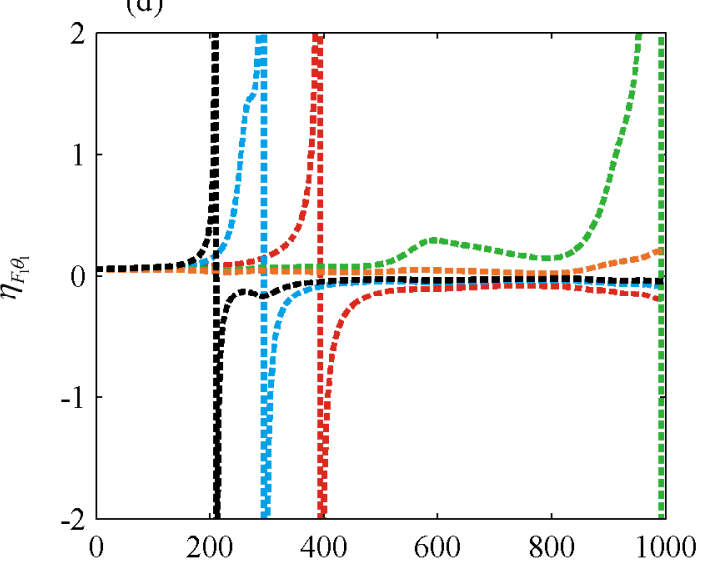

(f)

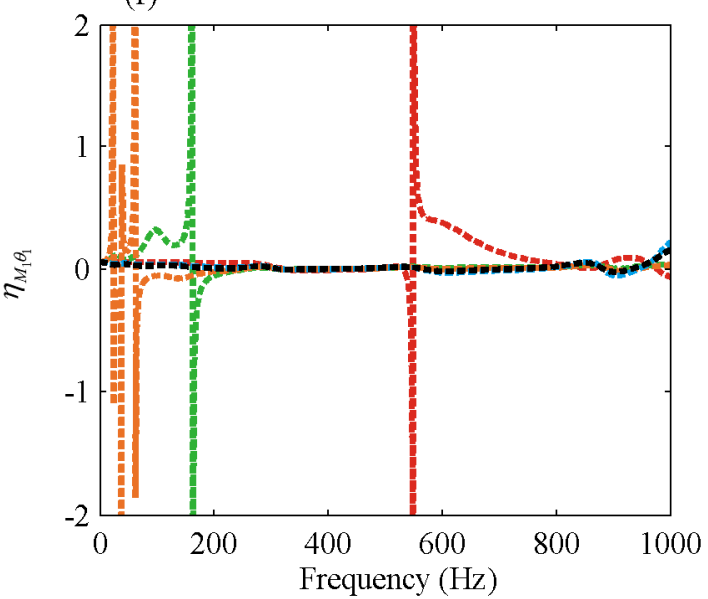

Figure 17: Inverse stiffness magnitude and loss factor for varied density of unconstrained structure. (a, b)

Translational, $(\mathbf{c}, \mathbf{d})$ coupling, and $(\mathbf{e}, \mathbf{f})$ rotational components: Key: $==-=, \rho_{o}=.98 \rho^{*} ;==-=, \rho_{o}=.99 \rho^{*} ;=-=-$, $\rho_{o}=\rho^{*},=-=-, \rho_{o}=1.01 \rho * ;-\cdots, \rho_{o}=1.02 \rho *$. 
(a)

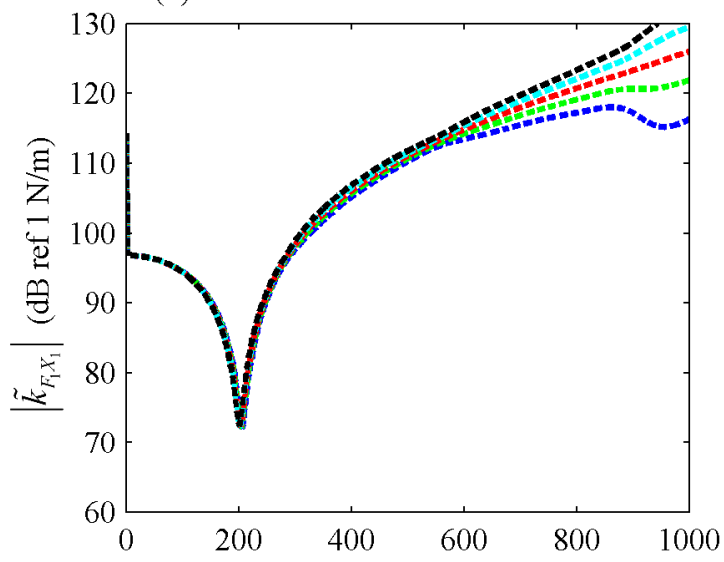

(c)

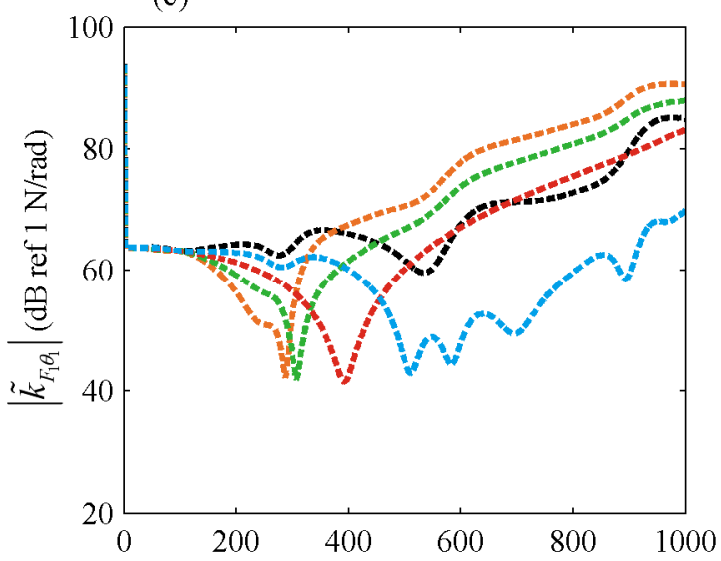

(e)

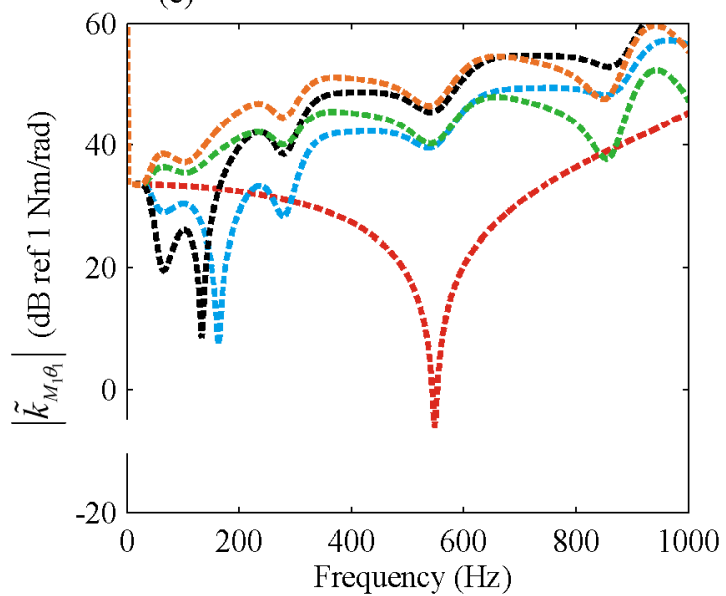

(b)

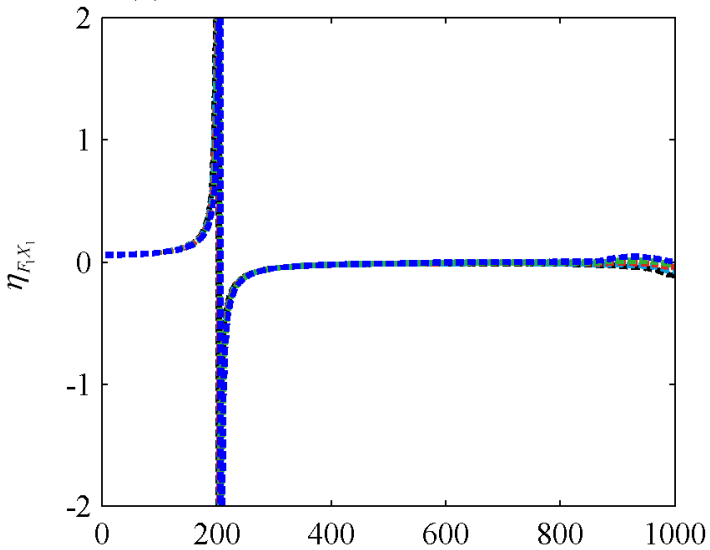

(d)
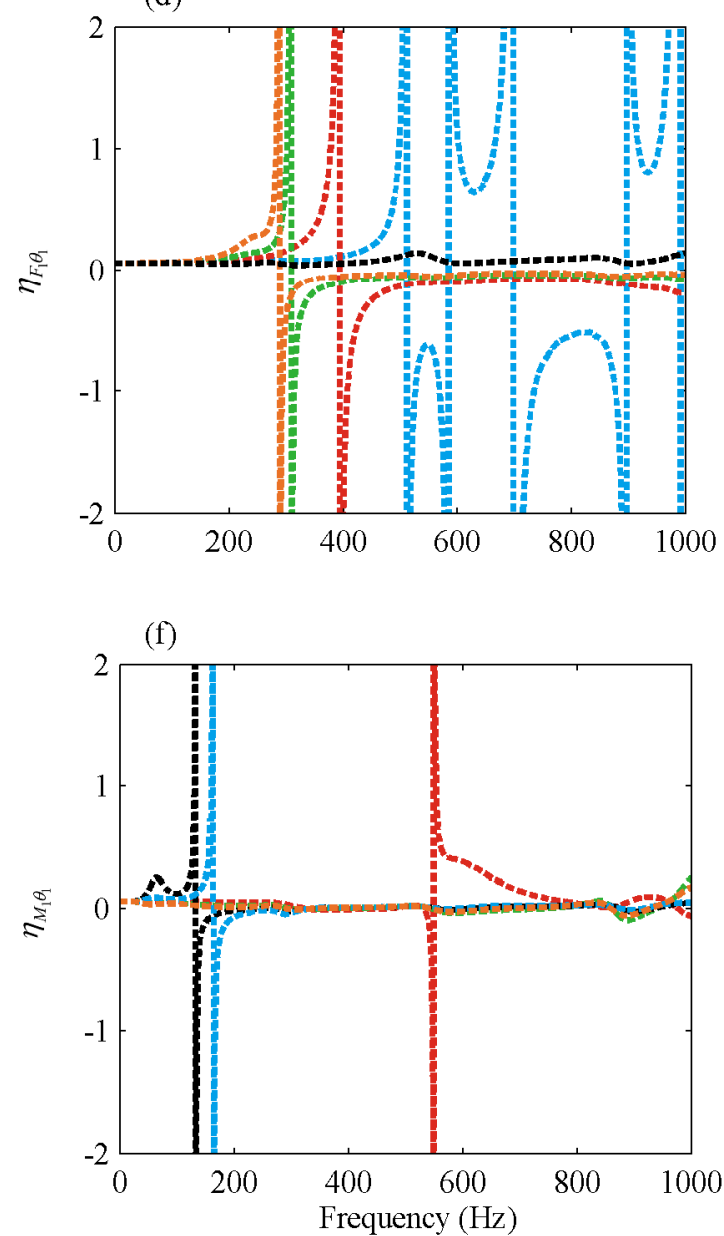

Figure 18 Inverse stiffness magnitude and loss factor for varied elastic modulus of unconstrained structure. (a, b) Translational, (c, d) coupling, and (e, f) rotational components: Key: = = =, $E_{o}=.98 E_{*}^{*} ;==-E_{o}=.99 E * ;=-=$ $=, E_{o}=E *,==-, E_{o}=1.01 E * ; \cdots, E_{o}=1.02 E$. 


\subsection{Experimental Validation of Proposed Method}

It is decided to only validate the inverse method procedure for the translational stiffness properties of the joints. In order to accomplish this, the number of observed and excited locations is increased to seven in order to minimized error due to conditioning (model 3 of Table 2). To obtain experimental compliance measurements, a roving impulse hammer technique is used to apply force inputs at the excited locations, and uniaxial accelerometers are located at the observed locations. The compliance frequency response functions are used in the estimation formula for unobserved compliance frequency response functions as $\boldsymbol{H}_{m e}^{*}$.

A large source of error in experiments may arise when measuring compliance frequency response functions. Since there are seven observed locations in the experiment, seven accelerometers are located on the constrained system. The accelerometers have a mass that may affect the identified results by altering the frequency response functions slightly. To illustrate this effect, a computational study is used. For one model, the mass and inertia of the accelerometer is measured and added to the mass matrix of the computational model of the beam. In a second model, the mass and inertia of seven accelerometers are added to the corresponding element locations in the global mass matrix. A comparison of a driving point compliance frequency response function on the free-free computational beam is used to show how these models differ (Figure 19). An effort is made to account for the accelerometer mass and inertia in the global mass matrix of the computational model during experiments, but do to human error and difficulty of modeling the accelerometer inertia error still results. It is important to take this into consideration when analyzing the experimentally identified stiffness of the joints. 


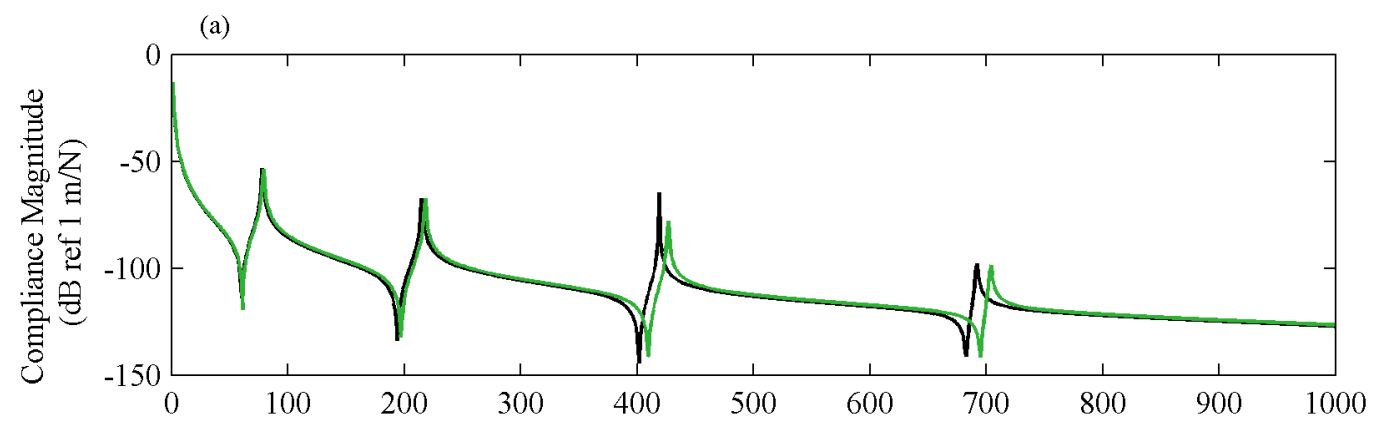

(b)

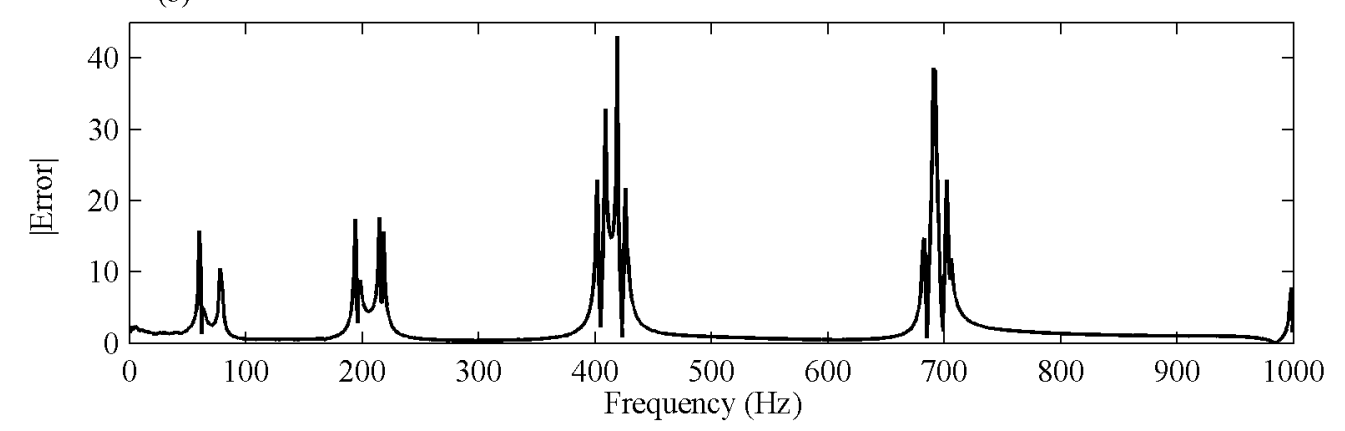

Figure 19: Mass effect due to accelerometer on calculated frequency response functions. (a) driving point compliance response and coordinate 2 (b) absolute error. Key: $\_, 1$ accelerometer attached to constrained system; $\stackrel{\longrightarrow}{\longrightarrow} 7$ accelerometers attached to constrained system.

The inverse experiment is validated two ways. First, the inverse identified stiffness properties will be compared to the verified stiffness properties from the past section. This is compared for a frequency range of 0-1000 Hz. Figure 21 illustrates the good agreement between the finite element and experimental inverse identified stiffness properties. Error occurs at low frequencies, but this is mostly due to dynamic ranging issues as well as incorrect modeling of elastomeric behavior. The simulated model assumes the elastomeric behavior exhibits structural damping throughout the entire frequency range. In reality, elastomers experience frequency dependence at low frequencies.

An interesting note about material B is that a second mode occurs at $950 \mathrm{~Hz}$. It is assumed that this is an internal joint mode. In order to verify this assumption, the FEA model of the fixed- 
free joint is checked for the displacement participation at $950 \mathrm{~Hz}, 150 \mathrm{~Hz}$ and a static test for material B as shown on Figure 20. At higher frequencies, the internal dynamics can no longer be approximated as a lumped spring element, as it has a mass associated with the material, resulting in an internal joint mode. The inverse identification procedure is able to successfully identify frequency dependent properties including joint modes.

\begin{tabular}{||c|c|c|}
\hline Static Stiffness & Mode 1 $(150 \mathrm{~Hz})$ & Mode 2 $(950 \mathrm{~Hz})$ \\
\hline \hline & & \\
\hline
\end{tabular}

Figure 20: Dynamic displacement of joint (enlarged deformation).

The second way to validate the inverse results is to compare it to the direct tests for low frequencies. Even though the direct measurement is a cross-point formulation, and the inverse measurement is a driving-point formulation, at low frequencies, a comparison between the two measurements is acceptable. This comparison is possible because at low frequencies the internal joint mass participation is negligible. Without the mass portion of the dynamic stiffness, the static stiffness should be the same at low frequencies. Again, due to dynamic ranging issues, it is difficult to obtain repeatable measurements for the lower frequency content $(0-20 \mathrm{~Hz})$, but at around $50 \mathrm{~Hz}$ the direct and inverse measurements start to converge on similar values (Figure 22). 
(a)

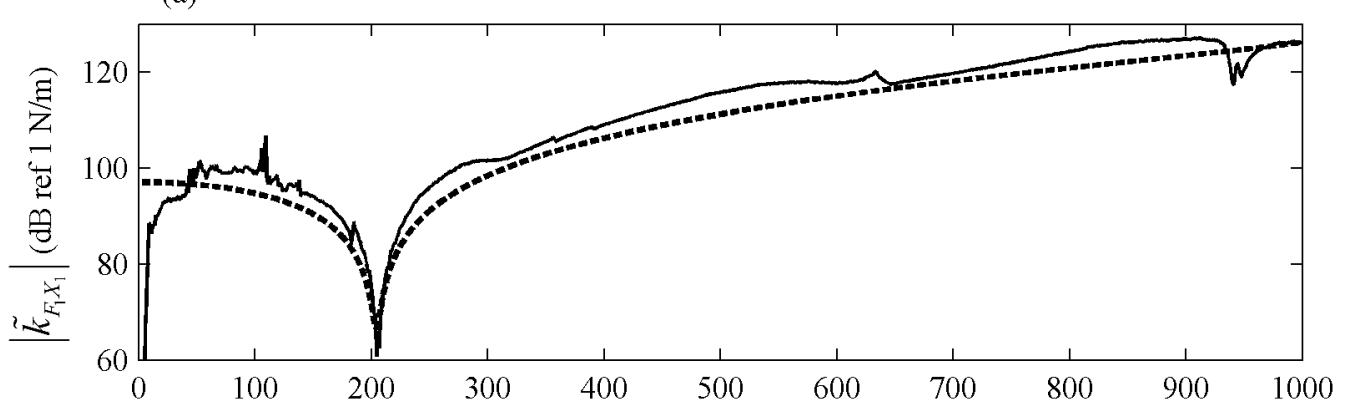

(b)
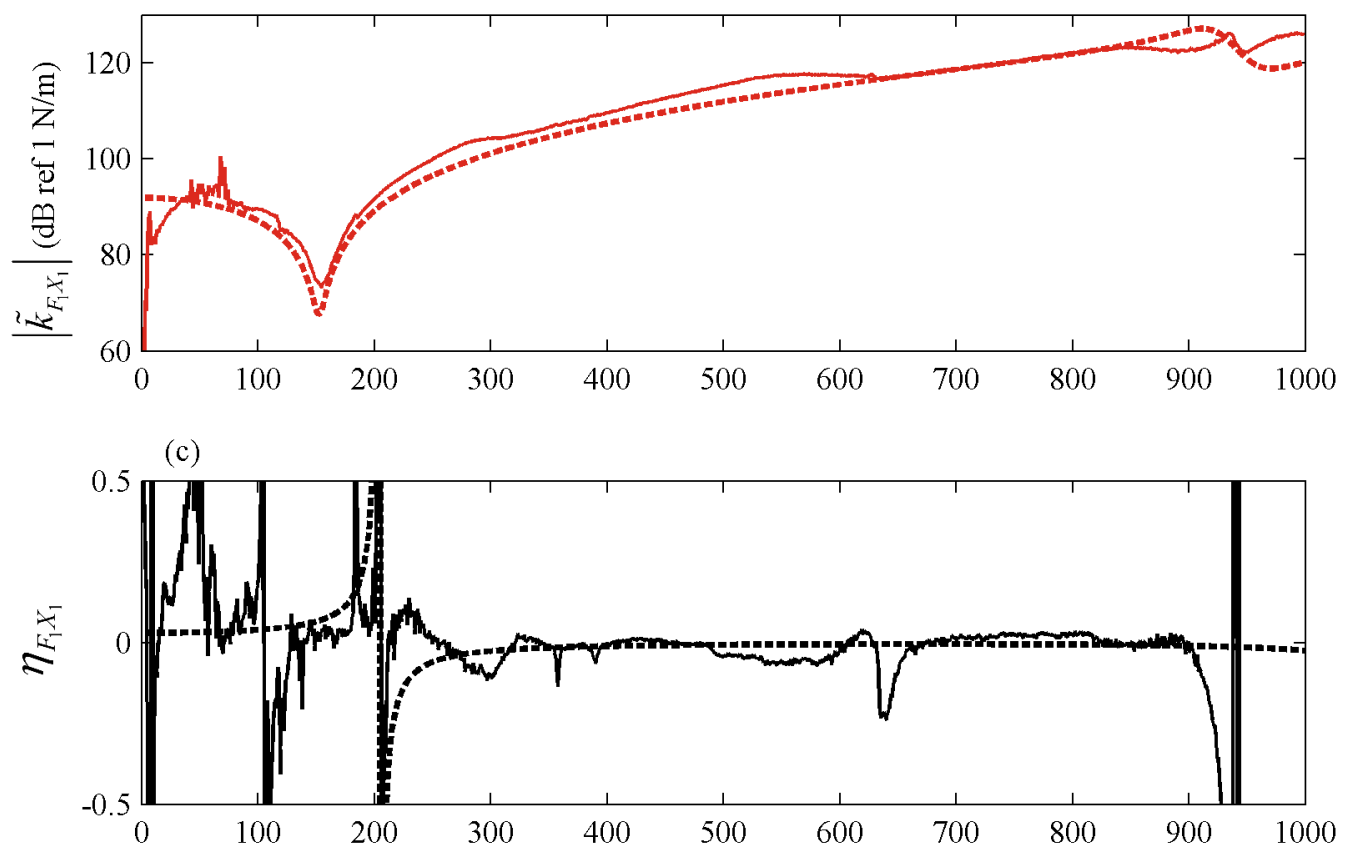

(d)

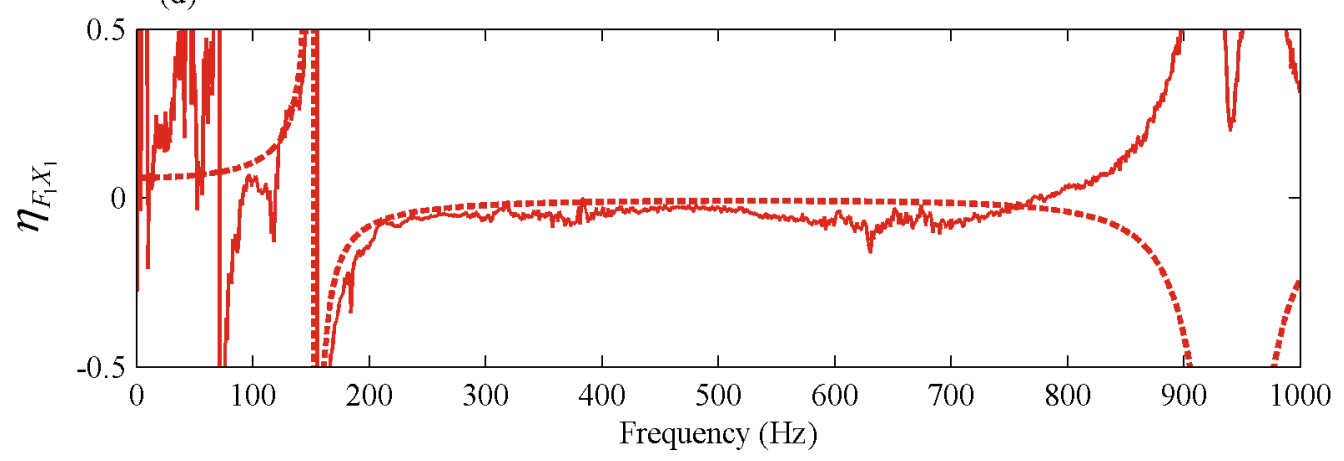

Figure 21: Comparison of inverse FEA and experimental identified dynamic stiffness magnitude (a, b) and loss factor (c, d). Key: __, Material A Experiment; - - - -, Material A FEA; __, Material B Experiment; - - - -, Material B FEA. 
(a)

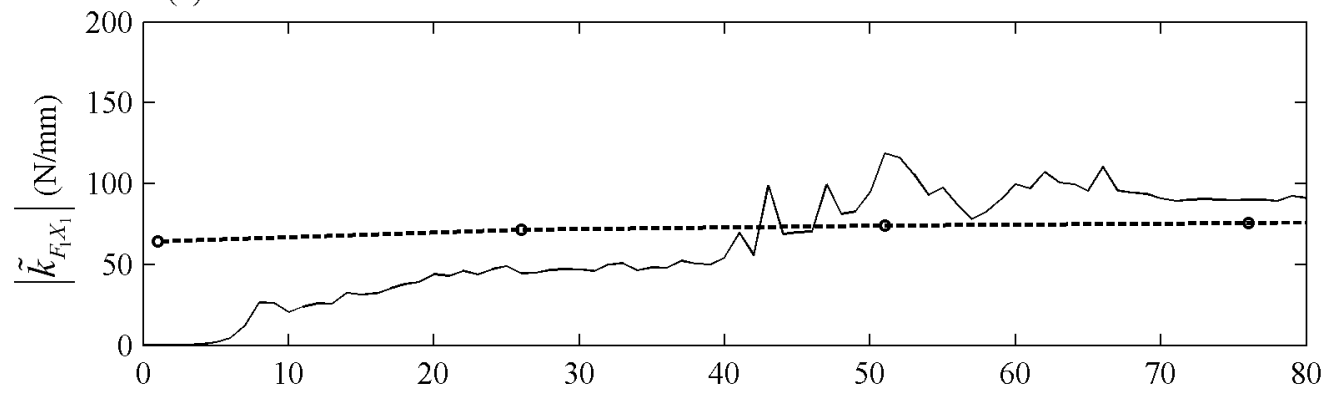

(b)

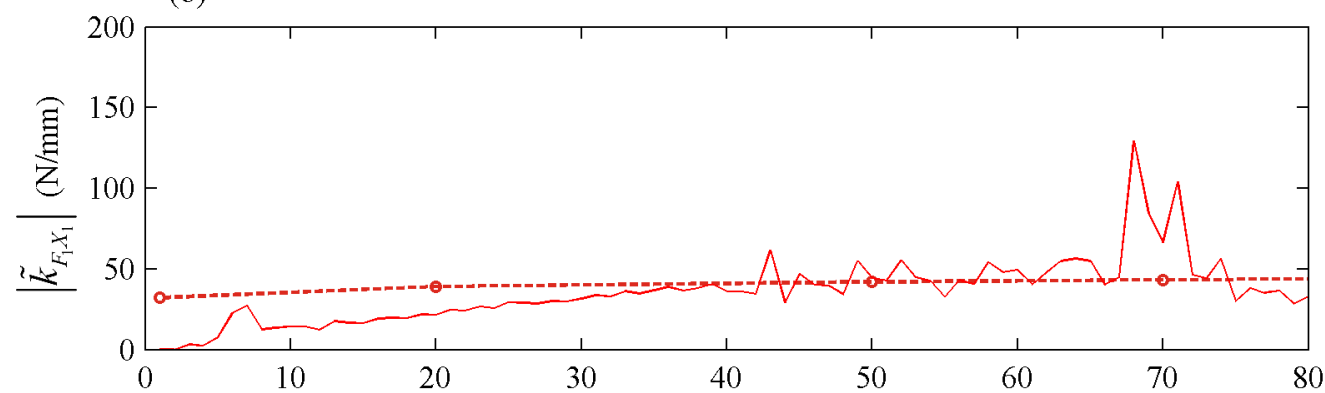

(c)

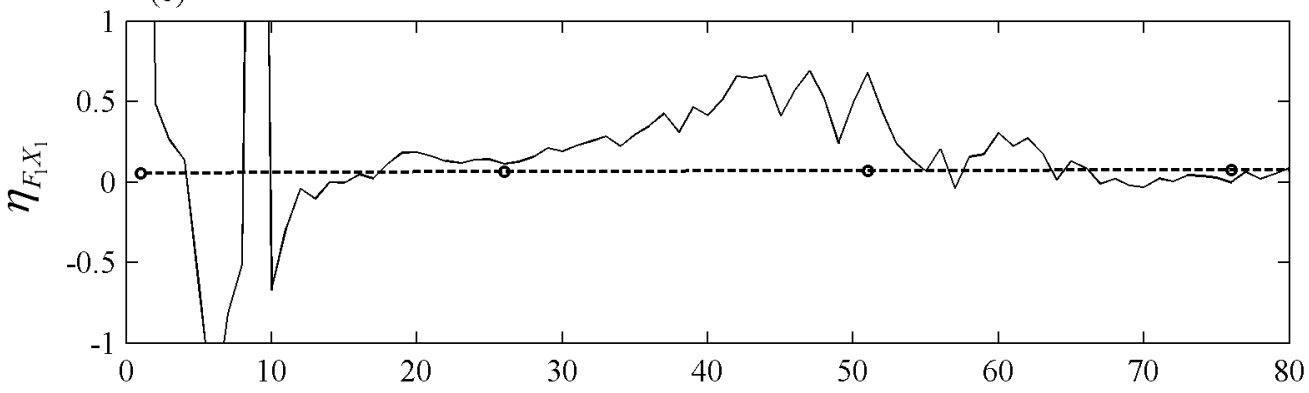

(d)

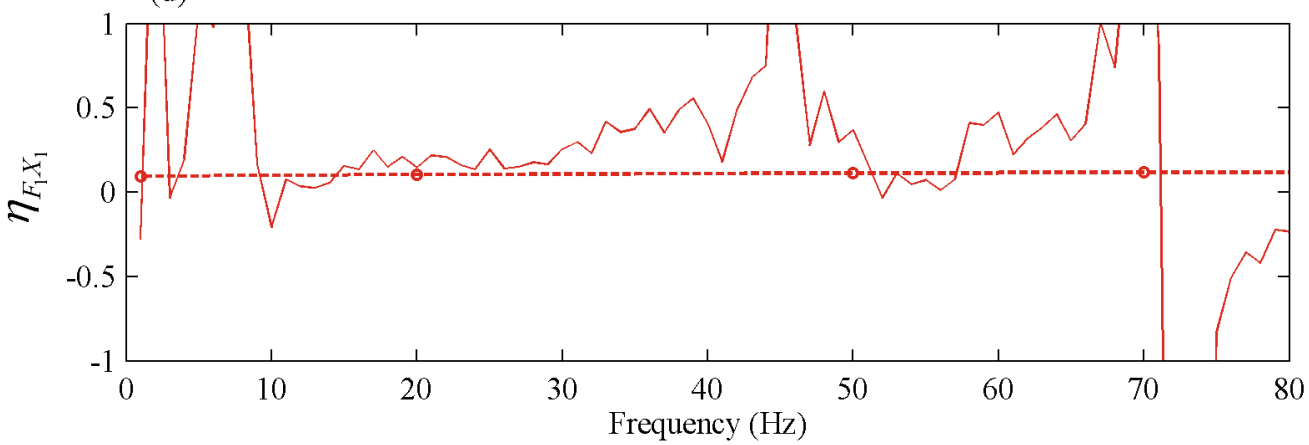

Figure 22: Comparisons of direct and inverse results for low frequency identified (a, b) dynamic stiffness magnitude and (c, d) loss factor. Key: (Material A) —_ Inverse Experiment; - - - - $\bigcirc$, Direct Experiment; (Material B) — Inverse Experiment; - - - - , Direct Experiment. 


\section{Chapter 5: Reduced Order Models to Include Internal Joint Resonance}

It is now of interest to see if a simple lumped parameter system can be used to approximate the behavior of the joint dynamic stiffness. This is completed for the verified results from the inverse procedure. For material A, it is noticed that one mode occurs throughout the frequency range. Since this is the case, a single degree of freedom system is used to approximate the behavior. The mass $(m)$, storage stiffness $\left(k^{\prime}\right)$, and loss factor $(\eta)$ are heuristically altered until similar agreement between the single degree of freedom and inverse FEA occur. The model for this lumped parameter system with the values for the parameters are shown on Figure 23a and Table 4. The equation of motion for the system at a given frequency is found as

$$
X=\frac{F}{\tilde{k}-m \omega^{2}} .
$$

where $X$ is the harmonic displacement of the mass, $m$, and $F$ is the harmonic excitation force acting on the block. The dynamic stiffness magnitude and loss factor of Equation (29) between the simplified lumped system and the FEA inverse identified results are shown on Figure 24. Figure 24 shows good agreement between the FEA and single degree of freedom system for the identified translational stiffness magnitude and loss factor.

The focus now is on the joint with elastic material B. Since material B displays two modes, a simple two degree of freedom model shown in Figure 23b is used to approximate this behavior. Again, values for the mass, stiffness and damping elements are heuristically altered until good agreement between the FEA and lumped system agree, displayed on Table 4. 
The two spring stiffness terms, $\tilde{k}$, are assumed to have the same value, and the model now consists of two masses, $m_{1}$ and $m_{2} . X_{1}$ and $X_{2}$ are the harmonic displacements associated with the masses. The equations of motion are solved for displacement $X_{1}$ due to a force acting on $m_{1}$. The equation of motion results in the following:

$$
X_{1}=\frac{\left(2 \tilde{k}-\omega^{2} m_{2}\right) F}{\left(\tilde{k}-\omega^{2} m_{1}\right)\left(2 \tilde{k}-m_{2} \omega^{2}\right)-(-\tilde{k})^{2}} \text {. }
$$

The dynamic stiffness magnitude and the loss factor of the lumped system approximating material B and the FEA Inverse method is displayed on Figure 24b and 24d. Again, the lumped parameter model shows good agreement with the identified magnitude stiffness and loss factor. These results are encouraging in that it allows for modeling of a widely known complex material (rubber) with simple discrete elements.

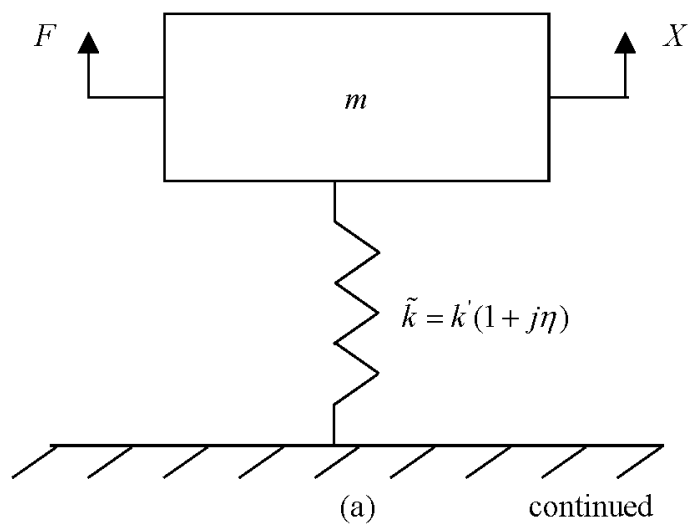

Figure 23: Lumped models to approximate joint behavior for a (a) single degree of freedom system approximation and (b) two degree of freedom system approximation. 
Figure 23 continued

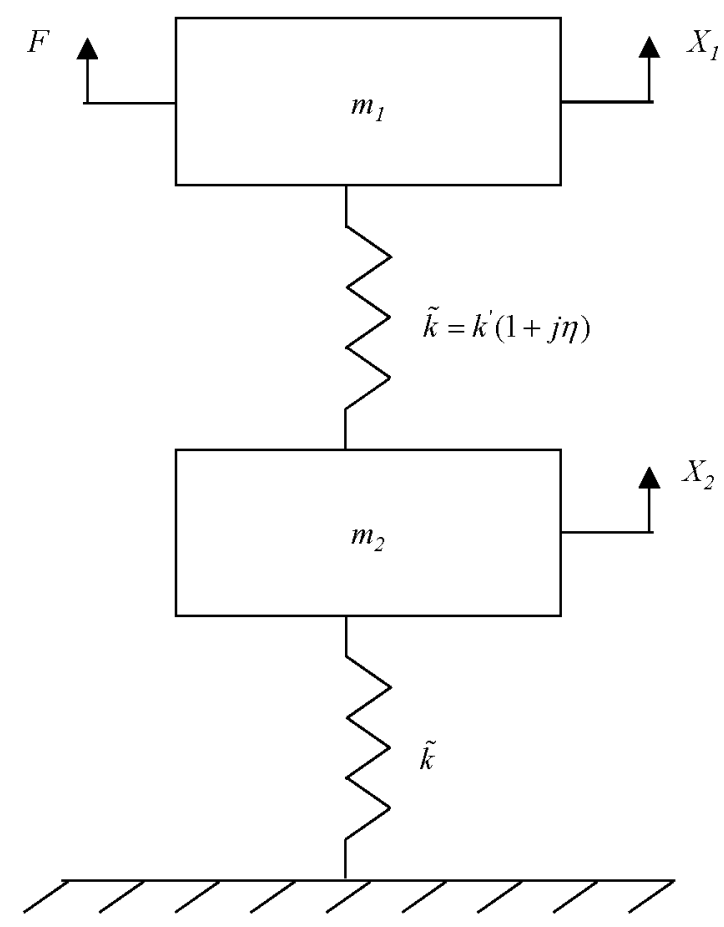

(b)

Table 4: Lumped models to approximate joint behavior.

\begin{tabular}{||c|c|c|c||}
\hline \hline Model & Parameters & Value & Unit \\
\hline \hline \multirow{3}{*}{ Figure 22a } & Mass, $m$ & 67.8 & $\mathrm{gm}$ \\
& Storage Stiffness, $k^{\prime}$ & 116.2 & $\mathrm{~N} / \mathrm{mm}$ \\
& Loss Factor, $\eta$ & 0.06 & - \\
\hline \hline \multirow{3}{*}{ Figure 23b } & Mass, $m_{1}$ & 50.6 & $\mathrm{gm}$ \\
& Mass, $m_{2}$ & 51.0 & $\mathrm{gm}$ \\
& Storage Stiffness, $k^{\prime}$ & 99 & $\mathrm{~N} / \mathrm{mm}$ \\
& Loss Factor, $\eta$ & 0.12 & - \\
\hline \hline
\end{tabular}


(a)

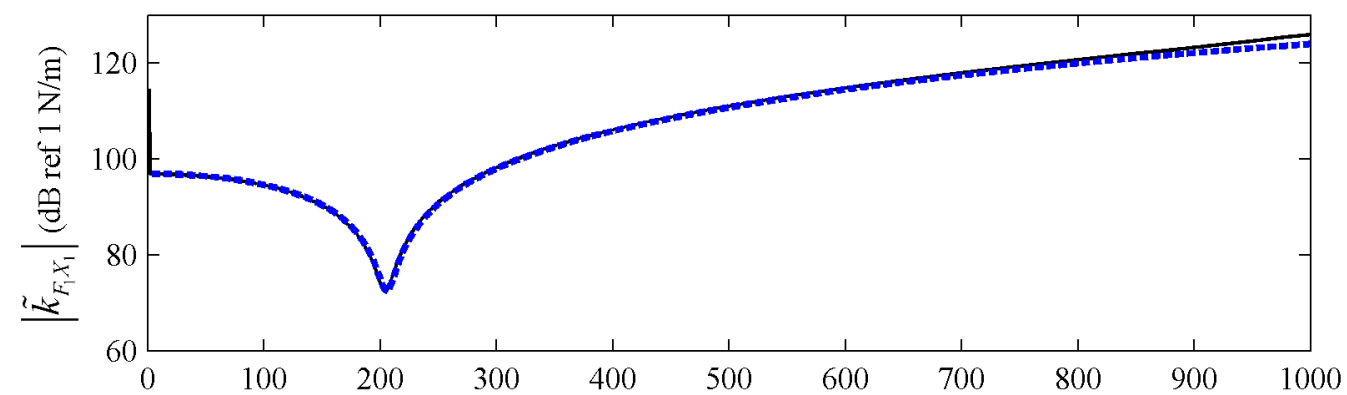

(b)

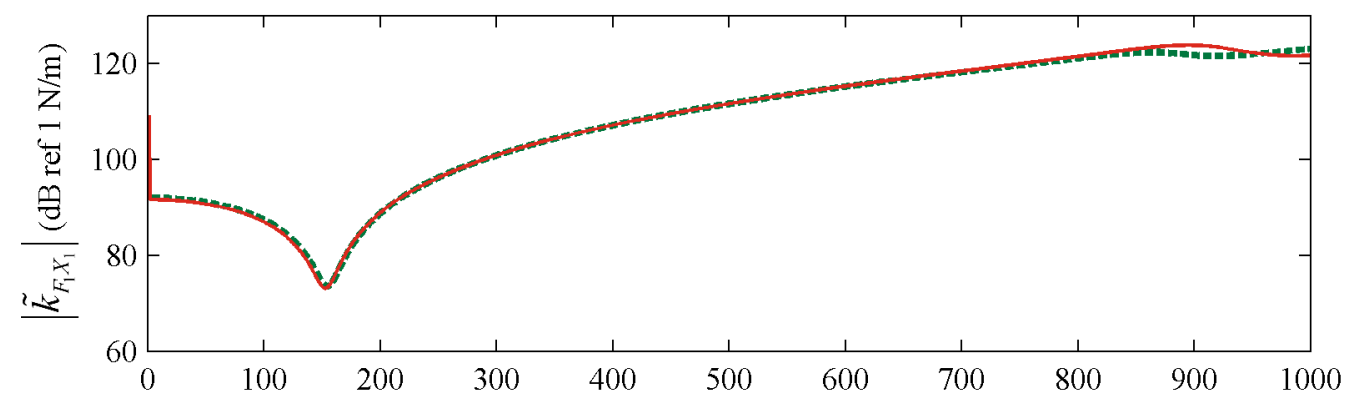

(c)

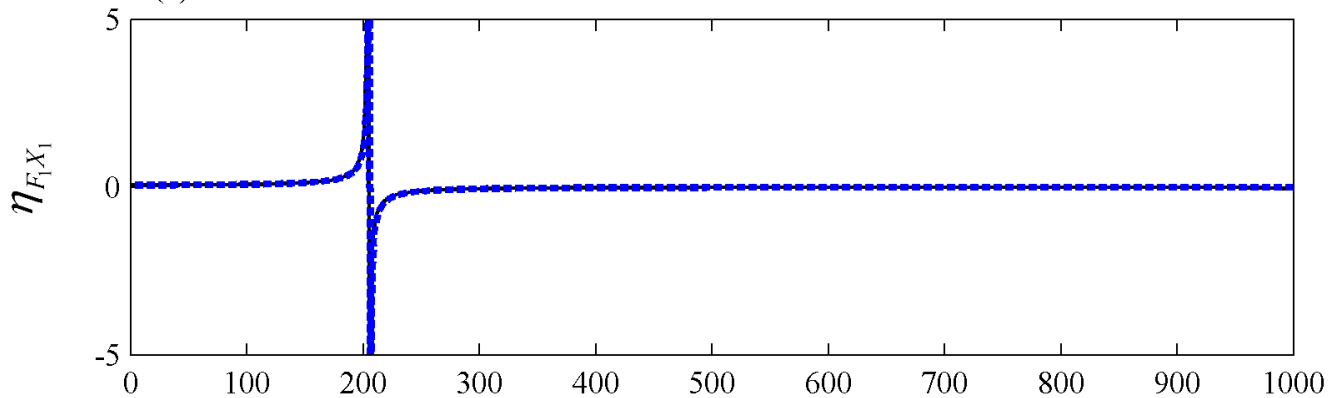

(d)

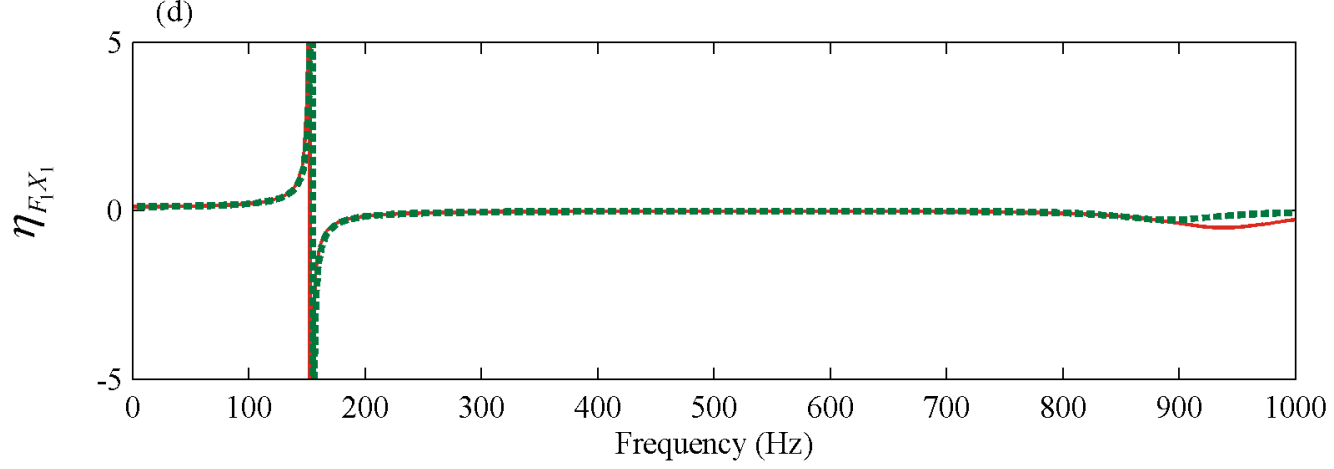

Figure 24: Comparison of lumped parameter approximations and inverse FEA showing the (a, b) translational stiffness magnitude and (c, d) translational loss factor. Key: ,__ Material A Inverse FEA; - - - -, Single Degree of Freedom System Model; ——, Material B Inverse FEA; - - - -, Two Degree of Freedom System Model. 


\section{Chapter 6: Conclusion}

\subsection{Summary}

The main focus of this study is to identify frequency dependent joint properties using inverse and direct methods. Good agreement between computational translational results of the two methods are seen for identified stiffness and loss factor. Experimental results show similar trends between the identified translational properties, but due to incorrect modeling and dynamic ranging issues, poor agreement occurs at certain frequencies. Experimental translational stiffness results showed good agreement with inverse simulated results as well as direct measurements at low frequencies. The inverse method is capable of identifying frequency dependent stiffness matrices for simulated data, but due to modeling sensitivity, identifying experimental rotational and coupling terms are left for future research. Finally, simple lumped parameter systems are shown to approximate the behavior of the elastomeric joints.

\subsection{Contributions}

The main contribution of this research is the design and analysis of new benchmark experiments. These new experiments are designed and constructed permitting a comparison between direct and inverse methods of identifying frequency dependent joint properties. Additionally, sources of error common to the inverse method are then examined and explained through finite element simulations. These sources of error consist of ill-conditioning of inversed matrices in the identification method as well as poor modeling of material properties associated with the constrained structure. This study has also shown the dynamics of a complicated 
elastomeric material with nonlinearities can still be approximated by simple lumped parameter systems. Lastly, this work provides physical insight into frequency dependent properties of elastomeric joints especially in the presence of internal joint resonances.

\subsection{Recommendations for Future Work}

Multidimensional properties are another important aspect of this inverse method. In order to obtain better identified rotational and coupling terms using the inverse identification method, incorporating rotational observed and excited locations should be considered. This could help improve the conditioning of the matrices in the identification method allowing for better accuracy of identified joint properties.

Another important concept to consider in future work, is the effect of preload. In many applications, joints may be under significant static preload during use. It is very important to model this preload to identify accurate stiffness measurements based on different operating ranges of the joint.

Different geometries should be explored to address ill-conditioning errors in the method. The method can be modified many ways by creating new structural geometries other than a simple beam element. In the end, this thesis shows the potential of this inverse identification procedure and future research can further determine whether this approach can be applicable in industrial settings. 


\section{References}

1. Kim, S., Singh, R, "Examination of High Frequency Characterization Methods for Mounts," SAE Transactions, Journal of Passenger Cars: Mechanical Systems, 110(6): 1625-1633, Paper \# 2001-01-1444, 2002.

2. MTS Systems, "Test Star II Control System," http://ametme.mnsu.edu/userfilesshared/DATA_ACQUISITION/mts/backup/New060904/ts2/docs/790_ 1x.pdf. Accessed Oct. 2014.

3. Holt, J., Rao, M., Blough, J., and Gruenberg, S., "Time history-based excitation in the dynamic characterization of automotive elastomers," Journal of Automobile Engineering, 221(3):271-284, 2007, doi: 10.1243/09544070JAUTO339.

4. MTS, "Elastomer Test Systems," http://mts.com/en/products/producttype/test-systems/loadframes-uniaxial/servohydraulic/elastomer/index.htm. Accessed Oct. 2014.

5. Noll, S., Dreyer, J., and Singh, R., "Comparative Assessment of Multi-Axis Bushing Properties Using Resonant and Non-Resonant Methods," SAE Int. J. Passeng. Cars - Mech. Syst. 6(2):1217-1223, 2013, doi: 10.4271/2013-01-1925.

6. Hong, S., Lee, C., "Identification of Linearised Joint Structural Parameters by Combined Use of Measured and Computed Frequency Response," Mechanical Systems and Signal Processing, 5(4):267-277, 1991, doi: 10.1016/0888-3270(91)90028-4.

7. Hong, S., Shamine, D., Shin, Y., "An In-situ Identification Method for Joint Parameters in Mechanical Structures," Journal of Vibration and Acoustics, 121(3):363-372, July 1999. doi: $10.1115 / 1.2893989$

8. Singh, R., "Automotive Noise and Vibration Control 1 With Lab Chapter 3 Volume 2 Course Notes," Columbus, 2013.

9. MathWorks, "MATLAB R2014a," The MathWorks Inc., Accessed August 2014.

10. Friedman, Z., Kosmatka, J., "An Improved Two-Node Timoshenko Beam Finite Element," Computers \& Structures, 47(3):473-481, 1993, doi: 10.1016/0045-7949(93)90243-7.

11. Meirovitch, L., Fundamentals of Vibration, (New York, McGraw-Hill, 2001), 238-239, ISBN 10: 0070413452 . 
12. Kosmatka, J. B., "An Improved Two-Node Finite Element for Stability and Natural Frequencies of Axial-Loaded Timoshenko Beams" Computers \& Structures, 57(1):141-149, 1995, doi: 10.1016/0045-7949(94)00595-T.

13. Lichtblau, D., Weisstein, E., "Condition Number." From MathWorld-A Wolfram Web Resource," October 2014. http://mathworld.wolfram.com/ConditionNumber.html. Accessed September 2014.

14. Colakoglue, M., Jerina, K., "Material Damping in 6061-T6511 Aluminum to Assess Fatigue Damage," Fatigue \& Fracture of Engineering Materials \& Structures, 26(1):79-84, January 2003, doi: 10.1046/j.1460-2695.2003.00603.x. 


\section{Appendix A: Static Force Deflection Curves for Joints}

Static force deflection curves were also found for the two joints. This plot shows that material A has a higher static stiffness value than material B. These measurements were taken on the direct testing fixture using the elastomer test system machine.

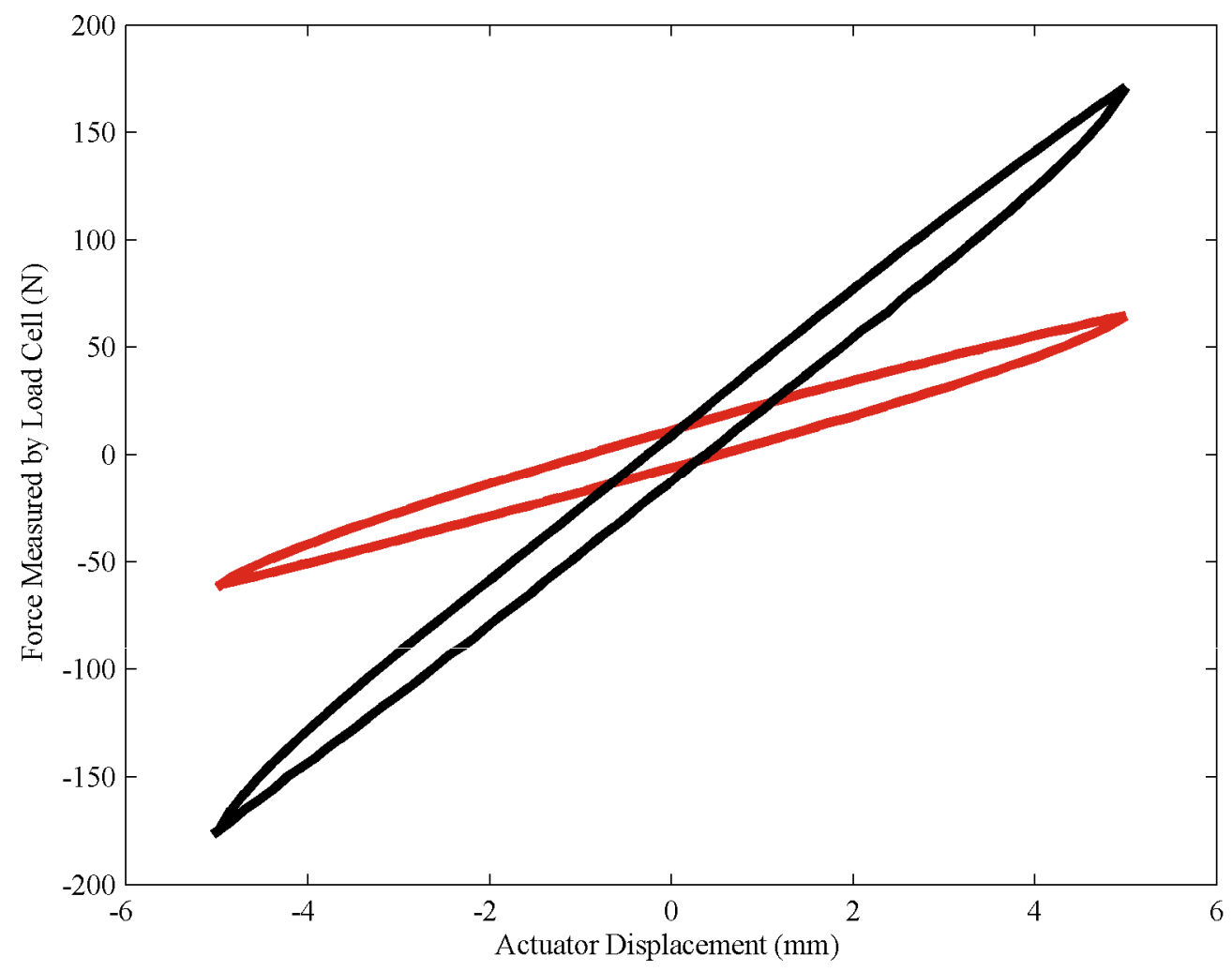

Figure 25: Static force deflection curves for two joints of different elastic materials. Key: (a) —, Material A; $\longrightarrow$, Material B. 